\title{
Module White Noise Calculus
}

\author{
L. ACCARDI, W. AYED, and H. OUERDIANE \\ Communicated by V. L. Girko
}

\begin{abstract}
Our main result is an infinitesimal characterization of Hilbert module module flows, not necessarily of inner type, in terms of stochastic derivations from the initial algebra into the Itô algebra. We prove that any such derivation is the difference of a $\star$-homomorphism and the trivial embedding.
\end{abstract}

Key words. Stochastic integration, Hilbert modules.

AMS classification. $60 \mathrm{H} 40,60 \mathrm{H} 35,46 \mathrm{~L} 55,58 \mathrm{~J} 65$.

\section{Introduction}

Stochastic integration on Hilbert modules was first developed in particular frameworks with the goal of solving concrete problems arising in the stochastic limit of QED (quantum electrodynamics) (see [4], [5]). Systematic developments of this calculus in more general frameworks were later developed by Lu [28], [29], [30], Speicher [35], Skeide [34], Goswami and Sinha [21].

To our knowledge the first nontrivial application of this calculus to a problem not originated from the module theory itself was the solution of the problem of explicitly constructing unitary stochastic equations driven by the square of white noise, obtained by Accardi and Boukas in [12]. A problem which could not be solved neither using the 1-st order representation of the 2-d order noise, obtained in [15], nor with more direct white noise methods [16]

A fall out of this construction was an elegant and simple (in particular coordinate free) representation, of the Itô algebra of stochastic differentials of a vector valued quantum noise, based on the notion of Hilbert module. This representation differs from the one previously obtained by Belavkin [19] without using Hilbert module techniques. The paper [11] however was essentially algebraic and the topological issues, concerning the extendability of the Itô table to the completion of the trivial Hilbert module, were left implicit.

The present paper fills this gap. Moreover it extends the Hilbert module approach to white noise flows not necessarily of inner type. It is interesting to notice that this extension leads to a purely algebraic notion of stochastic derivative thus clarifying the corresponding notion, introduced in [17] for operator valued measures.

Section 2 of this paper is devoted to generalize the scalar and the vector valued white noise in the module form beginning from the creation, annihilations and num- 
ber operator. In the third section, we give the module form of the white noise Itô table which combines the Fock space and the initial space. Using this, in Section 4, we give a simple proof of the unitary conditions based on this language. Section 5 is devoted to study flow equations and we find that the Hilbert module approach to these equations naturally leads to the notion of stochastic derivative.

In the last section, we prove that any such derivation is the difference of a $\star$-homomorphism and the trivial embedding.

\section{Notations}

\subsection{Trivial Hilbert modules}

In this section we recall some notations and known results on Hilbert modules, for all these and their proofs we refer to [33]. Let $\Gamma$ be a Hilbert space and let $\mathcal{B}_{S}$ be a $\mathbb{C}^{*}$-subalgebra of the algebra of all bounded operators on the Hilbert space $\mathcal{H}_{S}$. The algebraic tensor product:

$$
\mathcal{B}_{S} \otimes \Gamma
$$

has a natural structure of (trivial) right pre- $\mathcal{B}_{S}$-Hilbert module with inner product and right action given respectively by:

$$
\begin{gathered}
\left(b \otimes \psi \mid b_{1} \otimes \phi\right)=b^{*} b_{1}\langle\psi, \phi\rangle \in \mathcal{B}_{S} \\
(b \otimes \psi) b_{1}=b b_{1} \otimes \psi \in \mathcal{B}_{S} \otimes \Gamma
\end{gathered}
$$

where $b, b_{1} \in \mathcal{B}_{S}$ and $\psi, \phi \in \Gamma$. Completing it with respect to the norm

$$
\|x\|=\sqrt{\|(x \mid x)\|_{\infty}}, \forall x \in \mathcal{B}_{S} \otimes \Gamma
$$

where $\|\cdot\|_{\infty}$ is the $\mathbb{C}^{*}$-norm in $\mathcal{B}_{S}$, one obtains an Hilbert module denoted $\mathcal{H}$. Moreover, we will use the notation $\|x\|^{2}=\|x\|_{M}^{2}:=(x \mid x)$ for $x \in \mathcal{H}$. We recall from [33] the module form of the Cauchy-Schwarz inequality:

$$
|(y \mid x)|^{2} \leqslant\|(y \mid y)\|(x \mid x) ; x, y \in \mathcal{H}
$$

which implies

$$
\|(y \mid x)\|^{2} \leqslant\|(y \mid y)\|\|(x \mid x)\| ; x, y \in \mathcal{H}
$$

We will also use the same symbol $\mathcal{B}_{S} \otimes \Gamma$ to denote the completion of the algebraic tensor product with respect to the norm (2.3). On the trivial Hilbert module $\mathcal{B}_{S} \otimes \Gamma$, one can also define a left action of $\mathcal{B}_{S}$ by the formula

$$
b(c \otimes \psi):=b c \otimes \psi ; \quad b, c \in \mathcal{B}_{S} ; \psi \in \Gamma
$$


and this action is continuous for the norm (2.3). This induces the left action of $\mathcal{B}_{S} \otimes$ $\mathcal{B}(\Gamma)$ on $\mathcal{B}_{S} \otimes \Gamma$ defined by:

$$
\left(b_{S} \otimes b_{\Gamma}\right)\left(c_{S} \otimes \psi\right):=b_{S} c_{S} \otimes b_{\Gamma} \psi ; \quad b_{S}, c_{S} \in \mathcal{B}_{S} ; b_{\Gamma} \in \mathcal{B}(\Gamma) ; \psi \in \Gamma .
$$

Given any $\mathcal{B}_{S}$-Hilbert module $\mathcal{H}$, to any $\xi \in \mathcal{H}$ we associate right $\mathcal{B}_{S}$-linear map $\xi^{*}: \mathcal{H} \rightarrow \mathcal{B}_{S}$ defined through the identity $\xi^{*} \eta:=(\xi \mid \eta) ; \quad \xi^{*} \in \mathcal{H}^{*} ; \eta \in \mathcal{H}$. The set of all the $\xi^{*}(\xi \in \mathcal{H})$ is denoted $\mathcal{H}^{*}$ and is a left- $\mathcal{B}_{S}$-Hilbert module with inner product

$$
\left(\xi^{*} \mid \eta^{*}\right)_{\mathcal{H}^{*}}:=(\eta \mid \xi)_{\mathcal{H}} ; \quad \xi, \eta \in \mathcal{H}
$$

and left action given by

$$
b \xi^{*}:=\left(\xi b^{*}\right)^{*} ; \quad \xi \in \mathcal{H}, b \in \mathcal{B}_{S},
$$

$\mathcal{H}^{*}$ is called the dual Hilbert module of $\mathcal{H}$ (cf. [33]). If $\mathcal{B}_{1}$ is any $*$-algebra with a left action on $\mathcal{H}$, it has a natural right action on $\mathcal{H}^{*}$ given by:

$$
\left(\xi^{*} T\right) \eta:=\xi^{*}(T \eta)=(\xi \mid T \eta) ; \quad \xi, \eta \in \mathcal{H} ; T \in \mathcal{B}_{1}
$$

In particular, taking $\mathcal{B}_{1} \equiv \mathcal{B}_{S}$ with the action (2.6), we see that, if $x \in \mathcal{B}_{S}$ and $\xi \in$ $\mathcal{B}_{S} \otimes \Gamma$, then the product $\xi^{*} x$ is the element of $\left(\mathcal{B}_{S} \otimes \Gamma\right)^{*}$ defined as: $\xi^{*} x(y)=$ $(\xi \mid x y), \forall y \in \mathcal{B}_{S} \otimes \Gamma$. Moreover $x \xi^{*}(y):=(\xi \mid y x)$. In the case of a trivial Hilbert module $\mathcal{B}_{S} \otimes \Gamma$, there is also a left action of $\mathcal{B}(\Gamma)$ on $\mathcal{B}_{S} \otimes \Gamma$ given by linear extension of

$$
T(b \otimes \psi):=b \otimes T \psi ; b \in \mathcal{B}_{S}, \psi \in \Gamma, T \in \mathcal{B}(\Gamma)
$$

Lemma 2.1. For any $T \in \mathcal{B}(\Gamma)$ the operator defined by (2.8) is continuous and its norm is $\leqslant\|T\|_{\mathcal{B}(\Gamma)}$.

Proof. Let $F$ be a finite set and let $\sum_{j \in F} b_{j} \otimes \psi_{j} \in \mathcal{B}_{S} \otimes \Gamma$. Consider the identity:

$$
\left\|T\left(\sum_{j \in F} b_{j} \otimes \psi_{j}\right)\right\|_{M}^{2}=\sum_{j, k \in F}\left(b_{j} \otimes T \psi_{j} \mid b_{k} \otimes T \psi_{k}\right)=\sum_{j, k \in F} b_{j}^{*} b_{k}\left\langle T \psi_{j}, T \psi_{k}\right\rangle
$$

If $\varphi$ is any state on $\mathcal{B}_{S}$, the matrix $\left(\varphi\left(b_{j}^{*} b_{k}\right)\right)_{j, k \in F}$ is positive definite, hence there exists a finite set $\mathcal{G}$ and complex numbers $\lambda_{\alpha, j}, \alpha \in \mathcal{G}, j \in F$ such that $\varphi\left(b_{j}^{*} b_{k}\right)=$ $\sum_{\alpha \in \mathcal{G}} \overline{\lambda_{\alpha, j}} \lambda_{\alpha, k}$. Therefore,

$$
\begin{aligned}
\varphi\left(\left\|T\left(\sum_{j \in F} b_{j} \otimes \psi_{j}\right)\right\|_{M}^{2}\right) & =\sum_{\alpha \in \mathcal{G}} \sum_{j, k \in F} \overline{\lambda_{\alpha, j}} \lambda_{\alpha, k}\left\langle T \psi_{j}, T \psi_{k}\right\rangle=\sum_{\alpha \in \mathcal{G}}\left\|T\left(\sum_{j \in F} \lambda_{\alpha, j} \psi_{j}\right)\right\|_{\Gamma}^{2} \\
& \leqslant\|T\|^{2}\left\|\sum_{\alpha \in \mathcal{G}} \sum_{j \in F} \lambda_{\alpha, j} \psi_{j}\right\|_{\Gamma}^{2}=\|T\|^{2} \sum_{\alpha \in \mathcal{G}} \sum_{j, k \in F} \overline{\lambda_{\alpha, j}} \lambda_{\alpha, k}\left\langle\psi_{j}, \psi_{k}\right\rangle \\
& =\|T\|^{2} \sum_{j, k \in F} \varphi\left(b_{j}^{*} b_{k}\right)\left\langle\psi_{j}, \psi_{k}\right\rangle=\|T\|^{2} \varphi\left(\left\|\sum_{j \in F} b_{j} \otimes \psi_{j}\right\|_{M}^{2}\right)
\end{aligned}
$$


Since $\varphi$ is an arbitrary state on $\mathcal{B}_{S}$, this implies that:

$$
\left\|T\left(\sum_{j \in F} b_{j} \otimes \psi_{j}\right)\right\|_{M}^{2} \leqslant\|T\|_{\mathcal{B}(\Gamma)}\|\| \sum_{j \in F} b_{j} \otimes \psi_{j}\left\|^{2}\right\|_{\infty}
$$

and the statement follows.

Remark 2.2. The module norm topology induced by the norm (2.3) is the simplest topology on $\mathcal{B}_{S} \otimes \Gamma$ with the property described by the following lemma. Several other topologies can be considered on $\mathcal{B}_{S}$ but, since we will only consider equations with bounded coefficients, we can restrict our attention to the above mentioned one. Also, we will use the notation $|x|^{2}=x^{*} x, \quad \forall x \in \mathcal{B}_{S}$. The following facts are well known (see [33]).

Lemma 2.3. If $\left(e_{\alpha}\right)$ is any orthonormal basis of $\Gamma$, then any element of the trivial Hilbert module $\mathcal{B}_{S} \otimes \Gamma$, completed with the module norm topology (2.3), can be identified to a series $\sum_{\alpha} b_{S}^{\alpha} \otimes e_{\alpha}$ such that the series $\sum_{\alpha}\left|b_{S}^{\alpha}\right|^{2}$ converges in $\mathcal{B}_{S}$.

Remark 2.4. Any linear operator $b_{\Gamma}$ acting on $\Gamma$, will be identified to the operator $b_{\Gamma} \otimes i d_{\mathcal{H}_{S}}$, acting on $\mathcal{H}_{S} \otimes \Gamma \equiv \Gamma \otimes \mathcal{H}_{S}$. Thus: $b_{\Gamma}\left(f_{\Gamma} \otimes g_{S}\right):=\left(b_{\Gamma} f_{\Gamma}\right) \otimes g_{S} ; \quad f_{\Gamma} \in$ $\Gamma ; g_{S} \in \mathcal{H}_{S}$.

In the following, we will consider two main cases for $\Gamma$, namely:

$$
\Gamma:=L^{2}\left(\mathbb{R}^{d} ; \mathcal{K}\right) \quad, \quad \Gamma:=\Gamma\left(L^{2}\left(\mathbb{R}^{d} ; \mathcal{K}\right)\right)
$$

where $\mathcal{K}$ is an Hilbert space, $L^{2}\left(\mathbb{R}^{d} ; \mathcal{K}\right)$ is the Hilbert space of $\mathcal{K}$-valued functions on $\mathbb{R}^{d}$ with the inner product

$$
(f, g):=\int_{\mathbb{R}^{d}}\langle f(s), g(s)\rangle_{\mathcal{K}} d s \quad, \quad \forall f, g \in L^{2}\left(\mathbb{R}^{d} ; \mathcal{K}\right)
$$

and $\Gamma\left(L^{2}\left(\mathbb{R}^{d} ; \mathcal{K}\right)\right)$ denotes the bosonic Fock space over $L^{2}\left(\mathbb{R}^{d} ; \mathcal{K}\right)$ :

$$
\Gamma\left(L^{2}\left(\mathbb{R}^{d} ; \mathcal{K}\right)\right)=\oplus_{n=o}^{\infty} \otimes_{\text {sym }}^{n} L^{2}\left(\mathbb{R}^{d} ; \mathcal{K}\right)=\oplus_{n=0}^{\infty} \mathcal{F}_{n} \quad ; \quad \mathcal{F}_{0}:=\mathbb{C} \equiv \mathbb{C} \Phi
$$

Let $\mathcal{S}_{n}$ denote the permutation group on $n$ symbols. For any $n \in \mathbb{N}$ and for each $\pi \in \mathcal{S}_{n}$, the map

$$
\left(k_{1} \otimes \cdots \otimes k_{n}\right):=k_{\pi_{1}} \otimes \cdots \otimes k_{\pi_{n}} ; k_{1} \otimes \cdots \otimes k_{n} \in \otimes^{n} \mathcal{K}
$$

extends by linearity and continuity to a unitary operator $\pi_{\mathcal{K}}^{(n)}$ of $\otimes^{n} \mathcal{K}$ into itself. If

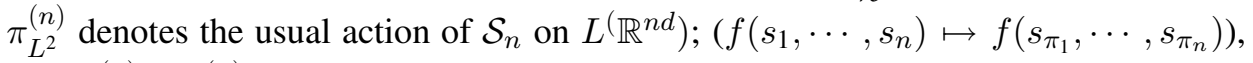
then $\pi_{L^{2}}^{(n)} \otimes \pi_{\mathcal{K}}^{(n)}$, defined by:

$$
(\pi \psi)\left(s_{1}, \cdots, s_{n}\right):=\pi_{\mathcal{K}}^{(n)} \psi\left(s_{\pi_{1}}, \cdots, s_{\pi_{n}}\right)
$$

acts on $\left(\otimes^{n} L^{2}\left(\mathbb{R}^{d}\right)\right) \otimes\left(\otimes^{n} \mathcal{K}\right) \equiv L^{2}\left(\mathbb{R}^{n d} ; \otimes^{n} \mathcal{K}\right)$. 
Definition 2.5. The subspace of $L^{2}\left(\mathbb{R}^{n d} ; \otimes^{n} \mathcal{K}\right)$, consisting of the fixed pints of all $\pi \in \mathcal{S}_{n}$ under the action (2.9), will be denoted $L_{\text {sym }}^{2}\left(\mathbb{R}^{n d} ; \otimes^{n} \mathcal{K}\right)$

Notice that $L_{\text {sym }}^{2}\left(\mathbb{R}^{n d} ; \otimes^{n} \mathcal{K}\right) \subseteq L_{\text {sym }}^{2}\left(\mathbb{R}^{n d}\right) \otimes\left(\otimes^{n} \mathcal{K}\right)$ where both spaces on the right side are identified to subspaces of $L^{2}\left(\mathbb{R}^{n d} ; \otimes^{n} \mathcal{K}\right)$.

Lemma 2.6. The natural embedding $\otimes^{n}(f \otimes k) \in \otimes_{\text {sym }}^{n} L^{2}\left(\mathbb{R}^{d} ; \mathcal{K}\right) \mapsto\left(\otimes^{n} f\right) \otimes$ $\left(\otimes^{n} k\right) \in L_{\text {sym }}^{2}\left(\mathbb{R}^{n d} ; \otimes^{n} \mathcal{K}\right)$ extends to a unitary isomorphism.

Proof. It is known that the elements of the form $\otimes^{n}(f \otimes \mathcal{K})$ are total in $\otimes_{\text {sym }}^{n} L^{2}\left(\mathbb{R}^{d} ; \mathcal{K}\right)$ and clearly the range of the above defined map is contained in $L_{\text {sym }}^{2}\left(\mathbb{R}^{n d} ; \otimes^{n} \mathcal{K}\right)$. Now suppose that the vector $\psi \in L_{\text {sym }}^{2}\left(\mathbb{R}^{n d} ; \otimes^{n} \mathcal{K}\right)$ is orthogonal to the closed linear span of the vectors $\left(\otimes^{n} f\right) \otimes\left(\otimes^{n} k\right) ;\left(f \in L^{2}\left(\mathbb{R}^{d}\right), k \in \mathcal{K}\right)$. Identifying $\psi$ with a function in its equivalence class, one then has

$0=\left\langle\left(\otimes^{n} f\right) \otimes\left(\otimes^{n} k\right), \psi\right\rangle=\int_{\mathbb{R}^{n d}}\left\langle\left(\otimes^{n} k\right), \psi\left(s_{1}, \cdots, s_{n}\right)\right\rangle_{\otimes^{n} \mathcal{K}} f\left(s_{1}\right) \cdots f\left(s_{n}\right) d s_{1} \cdots d s_{n}$

Thus $\left\langle\left(\otimes^{n} k\right), \psi\left(s_{1}, \cdots, s_{n}\right)\right\rangle_{\otimes^{n} \mathcal{K}}=0$ a.e. and, since the $\otimes^{n} k$ are total in $\otimes_{\text {sym }}^{n} \mathcal{K}$ and $\psi\left(s_{1}, \cdots, s_{n}\right) \in \otimes_{\text {sym }}^{n} \mathcal{K}$, we conclude that $\psi\left(s_{1}, \cdots, s_{n}\right)=0$ a.e. hence $\psi=0$ in $L^{2}$.

Corollary 2.7. The boson Fock space $\Gamma\left(L^{2}\left(\mathbb{R}^{d} ; \mathcal{K}\right)\right)$ is naturally isomorphic to $\bigoplus_{n \geq 0} L_{\text {sym }}^{2}\left(\mathbb{R}^{n d} ; \otimes^{n} \mathcal{K}\right),\left(L\left(\mathbb{R}^{0} ; \otimes^{0} \mathcal{K}\right):=\mathbb{C} \Phi\right)$.

Proof. This follows immediately from Lemma 2.6.

Definition 2.8. If $\mathcal{H}=\mathcal{B}_{S} \otimes \Gamma\left(L^{2}\left(\mathbb{R}^{d} ; \mathcal{K}\right)\right)$ and $\psi_{f} \in \Gamma\left(L^{2}\left(\mathbb{R}^{d} ; \mathcal{K}\right)\right)$ is an exponential vector, then any linear combination of elements of the form $c_{S} \otimes \psi_{f} \in \mathcal{B}_{S} \otimes$ $\Gamma\left(L^{2}\left(\mathbb{R}^{d} ; \mathcal{K}\right)\right)$ is called a module exponential vector. The set of module exponential vectors will be denoted $\mathcal{E}$.

By construction $\mathcal{E}$ can be identified to the set of all sums of the form $\sum_{\alpha \in F} b_{\alpha} \otimes \psi_{f_{\alpha}}$ where

(i) $F$ is a finite set.

(ii) $b_{\alpha} \in \mathcal{B}_{S}$.

(iii) $\left(\psi_{f_{\alpha}}\right), f_{\alpha} \in L^{2}\left(\mathbb{R}^{d} ; \mathcal{K}\right)$ arbitrary exponential vectors.

Definition 2.9. On the set $\mathcal{L}$ of linear adjointable operators with domain (of them and of their adjoint) containing $\mathcal{E} \subseteq \mathcal{H}_{S} \otimes \Gamma$ one defines the seminorms

$$
|X|_{\psi}:=\|X \psi\|^{2} ; \psi \in \mathcal{E}
$$




$$
|X|_{\psi, \varphi}:=|\langle\varphi, X \psi\rangle| ; \varphi, \psi \in \mathcal{E}
$$

The topology induced on $\mathcal{L}$ by the seminorms (2.10) (resp (2.11)) will be called the topology of strong (resp weak) convergence on the module exponential vectors.

Remark 2.10. $\mathcal{L}$ is total in $\mathcal{B}_{S} \otimes \Gamma\left(L^{2}\left(\mathbb{R}^{d} ; \mathcal{K}\right)\right)$ with respect the Hilbert module topology.

\subsection{Module creators}

The main point of the following three sections is to use Lemma (2.3) to give a meaning to expressions such as $b_{t}^{+}(F), b_{t}(G), b_{t}^{+} b_{t}(T)$ with

$$
F, G \in \mathcal{B}_{S} \otimes L^{2}\left(\mathbb{R}^{d} ; \mathcal{K}\right) ; \quad T \hat{\in} \mathcal{B}_{S} \otimes \mathcal{B}\left(L^{2}\left(\mathbb{R}^{d} ; \mathcal{K}\right)\right)
$$

To this goal we begin to define these quantities for elements in the algebraic tensor products

$F:=b_{S} \otimes \gamma, G:=c_{S} \otimes \gamma^{\prime} \in \mathcal{B}_{S} \otimes L^{2}\left(\mathbb{R}^{d} ; \mathcal{K}\right) ; T:=b_{S} \otimes b_{\Gamma} \hat{\in} \mathcal{B}_{S} \otimes \mathcal{B}\left(L^{2}\left(\mathbb{R}^{d} ; \mathcal{K}\right)\right)$

and then we extend the definition by continuity with respect to the topology given in Definition 2.9.

Let us recall our notations for the creation, annihilation and number operators on the Fock space $\Gamma\left(L^{2}\left(\mathbb{R}^{d} ; \mathcal{K}\right)\right)$ and the corresponding white noise densities.

For $g \in L^{2}\left(\mathbb{R}^{d} ; \mathcal{K}\right)$, the action of $b_{t}^{+}(g)$ on $\Gamma\left(L^{2}\left(\mathbb{R}^{d} ; \mathcal{K}\right)\right)$ is the operator valued distribution defined for a. e. $t \in \mathbb{R}^{d}$ by:

$$
\begin{aligned}
& b_{t}^{+}(g) \psi=\left(\left(b_{t}^{+}(g) \psi\right)^{(n)}\right):=\left(\left(b_{t}^{+}(g(t) \psi)^{(n)}\right)\right) ; \quad \forall \psi \in \Gamma\left(L^{2}\left(\mathbb{R}^{d} ; \mathcal{K}\right)\right) \\
&\left(b_{t}^{+}(g(t)) \psi\right)^{(n)}\left(s_{1}, \ldots, s_{n}\right) \\
& \quad=\frac{1}{\sqrt{n}} \sum_{i=1}^{i=n} \delta\left(t-s_{i}\right) g\left(s_{i}\right) \otimes \psi^{(n-1)}\left(s_{1}, \ldots, \hat{s}_{i}, \ldots, s_{n}\right), \text { a.e. }
\end{aligned}
$$

where $\delta(t-)$ is the Dirac measure. The creation and annihilation operators are defined for any square integrable measurable function $g \in L^{2}\left(\mathbb{R}^{d} ; \mathcal{K}\right)$ by:

$$
B_{g}^{+}=\int b_{t}^{+}(g(t)) d t
$$

and satisfy the commutation relation interpreted in the weak sense on the corresponding domains:

$$
\left[B_{f}, B_{g}^{+}\right]=\langle f, g\rangle_{L^{2}\left(\mathbb{R}^{d} ; \mathcal{K}\right)}, \quad B_{g} \Phi=0
$$


Definition 2.11. For any $c_{S} \otimes g \in \mathcal{B}_{S} \otimes L^{2}\left(\mathbb{R}^{d} ; \mathcal{K}\right) h_{S} \otimes \psi \in \mathcal{E}$,

$$
b_{t}^{+}\left(c_{S} \otimes g(t)\right)\left(h_{S} \otimes \psi\right)=c_{S} h_{S} \otimes b_{t}^{+}(g(t)) \psi, \quad \text { a.e. }
$$

Definition 2.12. For any $c_{S} \otimes f \in \mathcal{B}_{S} \otimes L^{2}\left(\mathbb{R}^{d} ; \mathcal{K}\right)$, define

$$
B^{+}\left(c_{S} \otimes f\right)\left(h_{S} \otimes \psi\right):=\left(c_{S} h_{S}\right) \otimes\left(B^{+}(f) \psi\right) \in \mathcal{H}_{S} \otimes \Gamma\left(L^{2}\left(\mathbb{R}^{d} ; \mathcal{K}\right)\right)
$$

where $h_{S} \in \mathcal{H}_{S}, \psi \in \operatorname{Dom}\left(B^{+}(f)\right) \subseteq \Gamma\left(L^{2}\left(\mathbb{R}^{d} ; \mathcal{K}\right)\right)$ and

$$
\begin{gathered}
\operatorname{Dom}\left(B^{+}(f)\right):= \\
\left\{\psi \in \Gamma\left(L^{2}\left(\mathbb{R}^{d} ; \mathcal{K}\right)\right): \sum_{n \geqslant 0}\left\|\sum_{i=1}^{n} f\left(s_{i}\right) \otimes \psi^{(n-1)}\left(s_{1}, \ldots, \hat{s_{i}}, \ldots, s_{n}\right)\right\|^{2}<\infty\right\}
\end{gathered}
$$

More explicitly, for each $n \geqslant 1$ :

$$
\begin{aligned}
& B^{+}\left(c_{S} \otimes f\right)\left(h_{S} \otimes \psi\right)^{(n)}\left(s_{1}, \ldots, s_{n}\right) \\
& =\frac{1}{\sqrt{n}} c_{S}\left(h_{S}\right) \otimes\left(\sum_{i=1}^{n} f\left(s_{i}\right) \otimes \psi^{(n-1)}\left(s_{1}, \ldots, \hat{s}_{i}, \ldots, s_{n}\right)\right), \text { a.e. }
\end{aligned}
$$

Remark 2.13. From Corollary 2.7, it is clear that the right hand side of (2.15) is in $\mathcal{B}_{S} \otimes \Gamma$. It follows that the domain of $\operatorname{Dom}\left(B^{+}\left(c_{S} \otimes f\right)\right)$ contains $\mathcal{E}_{+}(f):=\mathcal{E} \cap$ $\operatorname{Dom}\left(i d_{S} \otimes B^{+}(f)\right)=\mathcal{E}$.

Moreover, combining the equations (2.12) and (2.15), one can write, for any element $F \in \mathcal{B}_{S} \otimes L^{2}\left(\mathbb{R}^{d} ; \mathcal{K}\right)$ in the algebraic span of the vectors of the form $c_{S} \otimes f ; c_{S} \in$ $\mathcal{B}_{S}, f \in L^{2}\left(\mathbb{R}^{d} ; \mathcal{K}\right):$

$$
B^{+}(F)=\int b_{t}^{+}\left(F_{t}\right) d t
$$

Proposition 2.14. The map $c_{S} \otimes f \mapsto B^{+}\left(c_{S} \otimes f\right)$, from the algebraic Hilbert module $\mathcal{B}_{S} \otimes_{\text {alg }} L^{2}\left(\mathbb{R}^{d} ; \mathcal{K}\right)$ to the linear operators defined on the algebraic module exponential vectors can be extended by linearity and strong continuity on the module exponential vectors to the completion of the algebraic Hilbert module $\mathcal{B}_{S} \otimes_{\text {alg }} L^{2}\left(\mathbb{R}^{d} ; \mathcal{K}\right)$.

Proof. For any two elements $\beta, \beta_{1}$ of $L^{2}\left(\mathbb{R}^{d} ; \mathcal{K}\right)$, we use the notation $|\beta\rangle\left\langle\beta_{1}\right|$ for the operator on $L^{2}\left(\mathbb{R}^{d} ; \mathcal{K}\right)$ defined by

$$
|\beta\rangle\left\langle\beta_{1}\right|(x)=\left\langle\beta_{1}, x\right\rangle \beta, \quad \forall x \in L^{2}\left(\mathbb{R}^{d} ; \mathcal{K}\right)
$$


For any $F:=\sum_{j \in J} c_{S, j} \otimes f_{j} \in \mathcal{H}, \psi:=\sum_{\alpha \in K} h_{S, \alpha} \otimes \psi_{g_{\alpha}} \in \mathcal{E}_{+}(F)=\bigcap_{j \in K} \mathcal{E}_{+}\left(f_{j}\right)$ and $J, K$ finite sets, we have:

$$
\begin{aligned}
& \left\|B^{+}(F)\left(\sum_{\alpha \in K} h_{S, \alpha} \otimes \psi_{g_{\alpha}}\right)\right\|^{2} \\
= & \left\|B^{+}\left(\sum_{j \in J} c_{S, j} \otimes f_{j}\right)\left(\sum_{\alpha \in K} h_{S, \alpha} \otimes \psi_{g_{\alpha}}\right)\right\|^{2} \\
= & \left\|\sum_{j, \alpha} c_{S, j} h_{S, \alpha} \otimes B^{+}\left(f_{j}\right) \psi_{g_{\alpha}}\right\|^{2} \\
= & \sum_{j_{1}, \alpha_{1}} \sum_{j_{2}, \alpha_{2}}\left\langle c_{S, j_{1}} h_{S, \alpha_{1}}, c_{S, j_{2}} h_{S, \alpha_{2}}\right\rangle\left\langle B^{+}\left(f_{j_{1}}\right) \psi_{g_{\alpha_{1}}}, B^{+}\left(f_{j_{2}}\right) \psi_{g_{\alpha_{2}}}\right\rangle \\
= & \sum_{\alpha_{1}, \alpha_{2} \in K}\left\langle h_{S, \alpha_{1}}, \sum_{j_{1}, j_{2} \in J} c_{S, j_{1}}^{*} c_{S, j_{2}} h_{S, \alpha_{2}}\left\langle\psi_{g_{\alpha_{1}}},\left[B\left(f_{j_{1}}\right), B^{+}\left(f_{j_{2}}\right)\right] \psi_{\alpha_{\alpha_{2}}}\right\rangle\right\rangle \\
& -\sum_{\alpha_{1}, \alpha_{2} \in K}\left\langle h_{S, \alpha_{1}}, \sum_{j_{1}, j_{2} \in J} c_{S, j_{1}}^{*} c_{S, j_{2}} h_{S, \alpha_{2}}\right\rangle\left\langle B\left(f_{j_{1}}\right) \psi_{g_{\alpha_{1}}}, B\left(f_{j_{2}}\right) \psi_{g_{\alpha_{2}}}\right\rangle \\
= & J-I \leqslant \max \{J, I\}
\end{aligned}
$$

where

$$
\begin{aligned}
I & :=\sum_{\alpha_{1}, \alpha_{2} \in K}\left\langle h_{S, \alpha_{1}}, \sum_{j_{1}, j_{2} \in J} c_{S, j_{1}}^{*} c_{S, j_{2}} h_{S, \alpha_{2}}\right\rangle\left\langle B\left(f_{j_{1}}\right) \psi_{g_{\alpha_{1}}}, B\left(f_{j_{2}}\right) \psi_{g_{\alpha_{2}}}\right\rangle \\
& =\sum_{\alpha_{1}, \alpha_{2} \in K} \sum_{j_{1}, j_{2} \in J}\left\langle h_{S, \alpha_{1}}, c_{S, j_{1}}^{*} c_{S, j_{2}} h_{S, \alpha_{2}}\right\rangle\left\langle g_{\alpha_{1}}, f_{j_{2}}\right\rangle\left\langle f_{j_{1}}, g_{\alpha_{2}}\right\rangle\left\langle\psi_{g_{\alpha_{1}}}, \psi_{g_{\alpha_{2}}}\right\rangle \\
& =\sum_{\alpha_{1}, \alpha_{2} \in K}\left\langle\psi_{g_{\alpha_{1}}}, \psi_{g_{\alpha_{2}}}\right\rangle\left\langle h_{S, \alpha_{1}}, \sum_{j_{1}, j_{2} \in J} c_{S, j_{1}}^{*} c_{S, j_{2}}\left\langle g_{\alpha_{1}}, f_{j_{2}}\right\rangle\left\langle f_{j_{1}}, g_{\alpha_{2}}\right\rangle h_{S, \alpha_{2}}\right\rangle \\
& =\sum_{\alpha_{1}, \alpha_{2} \in K}\left\langle\psi_{g_{\alpha_{1}}}, \psi_{g_{\alpha_{2}}}\right\rangle\left\langle h_{S, \alpha_{1}},\left(\sum_{j_{1} \in J} c_{S, j_{1}}\left\langle f_{j_{1}}, g_{\alpha_{2}}\right\rangle\right)\left(\sum_{j_{2} \in J} c_{S, j_{2}}\left\langle g_{\alpha_{1}}, f_{j_{2}}\right\rangle\right) h_{S, \alpha_{2}}\right\rangle \\
& =\sum_{\alpha_{1}, \alpha_{2} \in K}\left\langle\psi_{g_{\alpha_{1}}}, \psi_{g_{\alpha_{2}}}\right\rangle\left\langle h_{S, \alpha_{1}},\left(F \mid\left(1 \otimes\left|g_{\alpha_{1}}\right\rangle\left\langle g_{\alpha_{2}}\right|\right) F\right) h_{S, \alpha_{2}}\right\rangle
\end{aligned}
$$

From Lemma (2.1) and from the Cauchy Schwartz inequality (2.4) for the Hilbert 
modules, it follows that, denoting $\|F\|$ the module norm (2.3) of $F$ :

$$
\begin{aligned}
|I| & \leqslant \sum_{\alpha_{1}, \alpha_{2} \in K}\left|\left\langle\psi_{g_{\alpha_{1}}}, \psi_{g_{\alpha_{2}}}\right\rangle\right| \cdot\left\|h_{S, \alpha_{1}}\right\| \cdot\left\|h_{S, \alpha_{2}}\right\|\left\|\left(F \mid\left(1 \otimes\left|g_{\alpha_{1}}\right\rangle\left\langle g_{\alpha_{2}}\right|\right) F\right)\right\|_{\infty} \\
& \left.\leqslant \sum_{\alpha_{1}, \alpha_{2} \in K}\left|\left\langle\psi_{g_{\alpha_{1}}}, \psi_{g_{\alpha_{2}}}\right\rangle\right| \cdot\left\|h_{S, \alpha_{1}}\right\| \cdot\left\|h_{S, \alpha_{2}}\right\|\|F\|^{\frac{1}{2}} \|\left(1 \otimes\left|g_{\alpha_{1}}\right\rangle\left\langle g_{\alpha_{2}}\right|\right) F\right) \|^{\frac{1}{2}} \\
& \leqslant\left(\sum_{\alpha_{1}, \alpha_{2} \in K}\left|\left\langle\psi_{g_{\alpha_{1}}}, \psi_{g_{\alpha_{2}}}\right\rangle\right| \cdot\left\|h_{S, \alpha_{1}}\right\| \cdot\left\|h_{S, \alpha_{2}}\right\| \|\left|g_{\alpha_{1}}\right\rangle\left\langle g_{\alpha_{2}}\right| \|^{\frac{1}{2}}\right)\|F\| \\
& \leqslant\left(\sum_{\alpha_{1}, \alpha_{2} \in K}\left|\left\langle\psi_{g_{\alpha_{1}}}, \psi_{g_{\alpha_{2}}}\right\rangle\right| \cdot\left\|h_{S, \alpha_{1}}\right\| \cdot\left\|h_{S, \alpha_{2}}\right\|\left|\left\langle g_{\alpha_{1}}, g_{\alpha_{2}}\right\rangle\right|^{\frac{1}{2}}\right)\|F\|
\end{aligned}
$$

Similarly

$$
\begin{aligned}
J & :=\sum_{\alpha_{1}, \alpha_{2}}\left\langle h_{S, \alpha_{1}}, \sum_{j_{1}, j_{2}} c_{S, j_{1}}^{*} c_{S, j_{2}} h_{S, \alpha_{2}}\left\langle\psi_{g_{\alpha_{1}}},\left[B\left(f_{j_{1}}\right), B^{+}\left(f_{j_{2}}\right)\right] \psi_{g_{\alpha_{2}}}\right\rangle\right\rangle \\
& =\sum_{\alpha_{1}, \alpha_{2}}\left\langle h_{S, \alpha_{1}}, \sum_{j_{1}, j_{2}} c_{S, j_{1}}^{*} c_{S, j_{2}} h_{S, \alpha_{2}}\left\langle\psi_{g_{\alpha_{1}}},\left\langle f_{j_{1}}, f_{j_{2}}\right\rangle \psi_{g_{\alpha_{2}}}\right\rangle\right\rangle \\
& =\sum_{\alpha_{1}, \alpha_{2}}\left\langle h_{S, \alpha_{1}} \otimes \psi_{g_{\alpha_{1}}}, \sum_{j_{1}} c_{S, j_{1}}^{*} \sum_{j_{1}} c_{S, j_{2}}\left\langle f_{j_{1}}, f_{j_{2}}\right\rangle h_{S, \alpha_{2}} \otimes \psi_{g_{\alpha_{2}}}\right\rangle \\
& =\left\langle\sum_{\alpha_{1}} h_{S, \alpha_{1}} \otimes \psi_{g_{\alpha_{1}}},(F \mid F) \sum_{\alpha_{2}} h_{S, \alpha_{2}} \otimes \psi_{g_{\alpha_{2}}}\right\rangle \\
& \leqslant\|(F \mid F)\|_{\infty}\left\|\sum_{\alpha} h_{S, \alpha} \otimes \psi_{g_{\alpha}}\right\|^{2}
\end{aligned}
$$

Finally we get with

$$
\begin{gathered}
c_{\psi}:=\max \left\{\sum_{\alpha_{1}, \alpha_{2} \in K}\left|\left\langle\psi_{g_{\alpha_{1}}}, \psi_{g_{\alpha_{2}}}\right\rangle\right| \cdot\left\|h_{S, \alpha_{1}}\right\| \cdot\left\|h_{S, \alpha_{2}}\right\|\left|\left\langle g_{\alpha_{1}}, g_{\alpha_{2}}\right\rangle\right|^{\frac{1}{2}}, \sum_{\alpha} h_{S, \alpha} \otimes \psi_{g_{\alpha}} \|^{2}\right\}: \\
\left\|B^{+}(F)(\psi)\right\|^{2} \leqslant c_{\psi}\|(F \mid F)\|_{\infty}
\end{gathered}
$$

The linearity of the map $F \mapsto B^{+}(F)$ and the inequality (2.19) imply that we extend by continuity the map $F \mapsto B^{+}(F)$ to the completion of the Hilbert module $\mathcal{B}_{S} \otimes$ $L^{2}\left(\mathbb{R}^{d} ; \mathcal{K}\right)$.

Notation: For every $c_{S} \in \mathcal{B}_{S}$ and $f \in L^{2}\left(\mathbb{R}^{d} ; \mathcal{K}\right)$, we define the operator valued measure on $\mathbb{R}^{d}$

$$
I \subseteq \mathbb{R}^{d} \mapsto B_{I}^{+}\left(c_{S} \otimes f\right):=B^{+}\left(c_{S} \otimes \chi_{I} \otimes f\right)
$$

For this measure, we will use the notation $d B_{t}^{+}\left(c_{S} \otimes f\right)$. 


\subsection{Module annihilators}

Recall that the annihilation white noise densities on the Fock space $\Gamma\left(L^{2}\left(\mathbb{R}^{d} ; \mathcal{K}\right)\right)$ are the operators defined, for any square integrable measurable function $g \in L^{2}\left(\mathbb{R}^{d} ; \mathcal{K}\right)$, by:

$$
B_{g}=\int b_{t}(g(t)) d t
$$

where $b_{t}(g(t))$ acts on $\mathcal{D}_{1}=\left\{\psi \in \Gamma\left(L^{2}\left(\mathbb{R}^{d} ; \mathcal{K}\right)\right) ; \sum_{n \geqslant 1} n\left\|\psi^{(n)}\right\|^{2}<\infty\right\}$ by:

$$
b_{t}(g) \psi=\left(\left(b_{t}(g) \psi\right)^{(n)}\right)=\left(\left(b_{t}(g(t)) \psi\right)^{(n)}\right) \text {, a.e. }
$$

Lemma 2.15. For every $c_{S} \in \mathcal{B}_{S}$ and $g \in L^{2}\left(\mathbb{R}^{d} ; \mathcal{K}\right)$, the adjoint of the operator $b_{t}^{+}\left(c_{S} \otimes g\right)\left(\right.$ resp. $\left.B^{+}\left(c_{S} \otimes g\right)\right)$ is well defined on $\mathcal{E}$ and is given for a. e. $t \in \mathbb{R}^{d}$ and $h_{S} \otimes \psi, h_{S}^{1} \otimes \phi \in \mathcal{E} \cap \mathcal{H}_{S} \otimes \mathcal{D}_{1}=\mathcal{E} b y$

$$
\begin{aligned}
& \left\langle b_{t}^{+}\left(c_{S} \otimes g\right)\left(h_{S} \otimes \psi\right), h_{S}^{1} \otimes \phi\right\rangle \\
= & \left\langle c_{S}\left(h_{S}\right) \otimes b_{t}^{+}(g(t))(\psi), h_{S}^{1} \otimes \phi\right\rangle \\
= & \left\langle c_{S}\left(h_{S}\right), h_{S}^{1}\right\rangle\left\langle b_{t}^{+}(g(t))(\psi), \phi\right\rangle=\left\langle h_{S}, c_{S}^{*}\left(h_{S}^{1}\right)\right\rangle\left\langle\psi, b_{t}(g(t))(\phi)\right\rangle \\
= & \left\langle h_{S} \otimes \psi, c_{S}^{*}\left(h_{S}^{1}\right) \otimes b_{t}(g(t))(\phi)\right\rangle=\left\langle h_{S} \otimes \psi, b_{t}\left(c_{S} \otimes g\right)\left(h_{S}^{1} \otimes \phi\right)\right\rangle
\end{aligned}
$$

Moreover, for any $c_{S} \otimes f \in \mathcal{B}_{S} \otimes_{\text {alg }} L^{2}\left(\mathbb{R}^{d} ; \mathcal{K}\right)$ and for any $h_{S} \otimes \psi, h_{S}^{1} \otimes \phi \in \mathcal{E}$ one has:

$$
\begin{aligned}
\left\langle B^{+}\left(c_{S} \otimes f\right)\left(h_{S} \otimes \psi\right), h_{S}^{1} \otimes \phi\right\rangle & =\left\langle c_{S}\left(h_{S}\right) \otimes B^{+}(f)(\psi), h_{S}^{1} \otimes \phi\right\rangle \\
& =\left\langle c_{S}\left(h_{S}\right), h_{S}^{1}\right\rangle\left\langle B^{+}(f)(\psi), \phi\right\rangle \\
& =\left\langle h_{S}, c_{S}^{*}\left(h_{S}^{1}\right)\right\rangle\langle\psi, B(f)(\phi)\rangle \\
& =\left\langle h_{S} \otimes \psi, c_{S}^{*}\left(h_{S}^{1}\right) \otimes B(f)(\phi)\right\rangle \\
& =\left\langle h_{S} \otimes \psi, B\left(c_{S} \otimes f\right)\left(h_{S}^{1} \otimes \phi\right)\right\rangle
\end{aligned}
$$

Definition 2.16. For any $c_{S} \otimes g \in \mathcal{B}_{S} \otimes L^{2}\left(\mathbb{R}^{d} ; \mathcal{K}\right)$, the operator $b_{t}\left(c_{S} \otimes g\right)$ will be called annihilator density and satisfies $\forall h_{S} \otimes \psi, h_{S}^{1} \otimes \phi$ module exponential vectors:

$$
\left\langle b_{t}^{+}\left(c_{S} \otimes g\right)\left(h_{S} \otimes \psi\right), h_{S}^{1} \otimes \phi\right\rangle=\left\langle h_{S} \otimes \psi, b_{t}\left(c_{S} \otimes g\right)\left(h_{S}^{1} \otimes \phi\right)\right\rangle
$$

One can conclude:

$$
b_{t}\left(c_{S} \otimes g\right)=c_{S}^{*} \otimes b_{t}(g)
$$

Remark 2.17. For any $c_{S} \otimes g \in \mathcal{B}_{S} \otimes L^{2}\left(\mathbb{R}^{d} ; \mathcal{K}\right), h_{S} \otimes \psi \in \mathcal{E}$, we define the annihilation densities $b_{t}(g(t))$ by the prescription

$$
b_{t}\left(c_{S} \otimes g\right)\left(h_{S} \otimes \psi\right):=c_{S}^{*}\left(h_{S}\right) \otimes b_{t}(g(t))(\psi), \text { a.e. }
$$

Then we extend it to the pre-Hilbert module $\mathcal{B}_{S} \otimes_{\text {alg }} L^{2}\left(\mathbb{R}^{d} ; \mathcal{K}\right)$ by linearity. 
Remark 2.18. We will use the same symbols $b_{t}^{+}(g), b_{t}(g)$ for the creation and annihilation densities acting on the Hilbert module, and on $\Gamma\left(L^{2}\left(\mathbb{R}^{d} ; \mathcal{K}\right)\right)$, so one has:

$$
b_{t}(g): \mathcal{H}_{S} \otimes \Gamma\left(L^{2}\left(\mathbb{R}^{d} ; \mathcal{K}\right)\right) \longrightarrow \mathcal{H}_{S} \otimes \Gamma\left(L^{2}\left(\mathbb{R}^{d} ; \mathcal{K}\right)\right)
$$

Moreover, $b_{t}(g)$ is well defined on $\mathcal{E}$.

Definition 2.19. For any $c_{S} \otimes f \in \mathcal{B}_{S} \otimes L^{2}\left(\mathbb{R}^{d} ; \mathcal{K}\right)$, the operator $B\left(c_{S} \otimes f\right)$ given in Lemma (2.15) will be called annihilator operator. From this, we get:

$$
B\left(c_{S} \otimes f\right)=c_{S}^{*} \otimes B(f)
$$

Proposition 2.20. The map $c_{S} \otimes f \mapsto B\left(c_{S} \otimes f\right)$, from the pre-Hilbert module $\mathcal{B}_{S} \otimes_{\text {alg }}$ $L^{2}\left(\mathbb{R}^{d} ; \mathcal{K}\right)$ to the linear operators defined on the module exponential vectors, can be extended by anti-linearity and strong continuity on the module exponential vectors to the completion of the whole Hilbert module $\mathcal{B}_{S} \otimes L^{2}\left(\mathbb{R}^{d} ; \mathcal{K}\right)$.

Proof. The anti-linearity of the map $F \mapsto B(F)$ is clear from (2.23) and (2.20). If a sequence $\left(F_{n}\right) \in \mathcal{B}_{S} \otimes L^{2}\left(\mathbb{R}^{d} ; \mathcal{K}\right)$ converges to 0 with respect to the Hilbert module topology, then $B(F)$ converges to 0 in the strong topology on $\mathcal{E}$. Moreover, for any

$$
F=\sum_{j \in I_{1}} c_{S, j} \otimes f_{j} \in \mathcal{B}_{S} \otimes L^{2}\left(\mathbb{R}^{d} ; \mathcal{K}\right)
$$

and

$$
\psi=\sum_{\alpha \in I_{2}} h_{S, \alpha} \otimes \psi_{g_{\alpha}} \in \mathcal{E}
$$

$\left(I_{1}, I_{2}\right.$ are finite sets)

$$
\begin{aligned}
\|B(F) \psi\|^{2} & =\left\|B\left(\sum_{j} c_{S, j} \otimes f_{j}\right)\left(\sum_{\alpha \in \text { Ifinite }} h_{S, \alpha} \otimes \psi_{g_{\alpha}}\right)\right\|^{2} \\
& =\left\|\sum_{j, \alpha} c_{S, j} h_{S, \alpha} \otimes B\left(f_{j}\right) \psi_{g_{\alpha}}\right\|^{2} \\
& =\sum_{j_{1}, \alpha_{1}} \sum_{j_{2}, \alpha_{2}}\left\langle c_{S, j_{1}} h_{S, \alpha_{1}}, c_{S, j_{2}} h_{S, \alpha_{2}}\right\rangle\left\langle B\left(f_{j_{1}}\right) \psi_{g_{\alpha_{1}}}, B\left(f_{j_{2}}\right) \psi_{g_{\alpha_{2}}}\right\rangle \\
& =\sum_{\alpha_{1}, \alpha_{2}} \sum_{j_{1}, j_{2}}\left\langle h_{S, \alpha_{1}}, c_{S, j_{1}}^{*} c_{S, j_{2}} h_{S, \alpha_{2}}\right\rangle\left\langle g_{\alpha_{1}}, f_{j_{2}}\right\rangle\left\langle f_{j_{1}}, g_{\alpha_{2}}\right\rangle\left\langle\psi_{g_{\alpha_{1}}}, \psi_{g_{\alpha_{2}}}\right\rangle \\
& =\sum_{\alpha_{1}, \alpha_{2}}\left\langle\psi_{g_{\alpha_{1}}}, \psi_{g_{\alpha_{2}}}\right\rangle\left\langle h_{S, \alpha_{1}}, \sum_{j_{1}, j_{2}} c_{S, j_{1}}^{*} c_{S, j_{2}}\left\langle g_{\alpha_{1}}, f_{j_{2}}\right\rangle\left\langle f_{j_{1}}, g_{\alpha_{2}}\right\rangle h_{S, \alpha_{2}}\right\rangle \\
& =\sum_{\alpha_{1}, \alpha_{2}}\left\langle\psi_{g_{\alpha_{1}}}, \psi_{g_{\alpha_{2}}}\right\rangle\left\langle h_{S, \alpha_{1}},\left(F \mid\left(1 \otimes\left|g_{\alpha_{1}}\right\rangle\left\langle g_{\alpha_{2}}\right|\right) F\right) h_{S, \alpha_{2}}\right\rangle
\end{aligned}
$$


The thesis now follows from the inequality (2.18). The same argument as in Proposition (2.14) leads to the inequality

$$
\|B(F) \psi\| \leqslant c^{\prime}{ }_{\psi}\|(F \mid F)\|
$$

where $c_{\psi}^{\prime}:=\sum_{\alpha_{1}, \alpha_{2} \in K}\left|\left\langle\psi_{g_{\alpha_{1}}}, \psi_{g_{\alpha_{2}}}\right\rangle\right| .\left\|h_{S, \alpha_{1}}\right\| .\left\|h_{S, \alpha_{2}}\right\|\left|\left\langle g_{\alpha_{1}}, g_{\alpha_{2}}\right\rangle\right|^{\frac{1}{2}}$ and from this the required strong continuity follows.

Notation: For every $c_{S} \in \mathcal{B}_{S}$ and $f \in L^{2}\left(\mathbb{R}^{d} ; \mathcal{K}\right)$, we define the operator valued measure on $\mathbb{R}^{d}$

$$
I \subseteq \mathbb{R}^{d} \mapsto B_{I}\left(c_{S} \otimes f\right)=B\left(c_{S} \otimes \chi_{I} \otimes f\right)
$$

We will use the notation $d B_{t}\left(c_{S} \otimes f\right)$ for this measure.

\subsection{Module number operators}

Lemma 2.21. If the series

$$
T=\sum_{j}\left|f_{j}\right\rangle\left\langle g_{j}\right| \in \mathcal{B}\left(L^{2}\left(\mathbb{R}^{d}, \mathcal{K}\right)\right), \quad f_{j}, g_{j} \in L^{2}\left(\mathbb{R}^{d}, \mathcal{K}\right)
$$

converge weakly in $L^{2}\left(\mathbb{R}^{d}, \mathcal{K}\right)$, then the series

$$
n\left(t, \sum_{j}\left|f_{j}\right\rangle\left\langle g_{j}\right|\right):=\sum_{j} b_{t}^{+}\left(f_{j}(t)\right) b_{t}\left(g_{j}(t)\right), \text { a.e. }
$$

weakly on the module exponential vectors.

Proof. For all $T \in B\left(L^{2}\left(\mathbb{R}^{d}, \mathcal{K}\right)\right)$ of the form (2.25) and for any pair of exponential vectors $\psi_{\alpha}, \psi_{\beta} \in \Gamma\left(L^{2}\left(\mathbb{R}^{d}, \mathcal{K}\right)\right), \alpha, \beta \in L^{2}\left(\mathbb{R}^{d}, \mathcal{K}\right)$, one has for a. e. $t \in \mathbb{R}^{d}$ :

$$
\begin{aligned}
\left\langle\psi_{\alpha}, n\left(t, \sum_{j}\left|f_{j}\right\rangle\left\langle g_{j}\right|\right) \psi_{\beta}\right\rangle & =\sum_{j}\left\langle\psi_{\alpha}, b_{t}^{+}\left(f_{j}(t)\right) b_{t}\left(g_{j}(t)\right) \psi_{\beta}\right\rangle \\
& =\sum_{j}\left\langle\alpha(t), f_{j}(t)\right\rangle\left\langle g_{j}(t), \beta(t)\right\rangle\left\langle\psi_{\alpha}, \psi_{\beta}\right\rangle \\
& =\left\langle\alpha(t),\left(\sum_{j}\left|f_{j}(t)\right\rangle\left\langle g_{j}(t)\right|\right) \beta(t)\right\rangle\left\langle\psi_{\alpha}, \psi_{\beta}\right\rangle
\end{aligned}
$$

Notation: Denote by $\mathcal{T}$ the closure of the space spanned by operators of the form (2.25) with respect the weak topology on $B\left(L^{2}\left(\mathbb{R}^{d}, \mathcal{K}\right)\right)$. 
Definition 2.22. For any linear operator $T \in \mathcal{T}$

$$
T=\sum_{j}\left|f_{j}\right\rangle\left\langle g_{j}\right| \in \mathcal{B}\left(L^{2}\left(\mathbb{R}^{d}, \mathcal{K}\right)\right), \quad f_{j}, g_{j} \in L^{2}\left(\mathbb{R}^{d}, \mathcal{K}\right)
$$

where the series converges weakly on $L^{2}\left(\mathbb{R}^{d} ; \mathcal{K}\right)$, the number density $n(t, T)$ is defined by:

$$
n\left(t, \sum_{j}\left|f_{j}\right\rangle\left\langle g_{j}\right|\right):=\sum_{j} b_{t}^{+}\left(f_{j}(t)\right) b_{t}\left(g_{j}(t)\right), \text { a.e. }
$$

where the series converges weakly on the module exponential vectors.

Definition 2.23. Let $T=\sum_{j} c_{S, j} \otimes T_{j} \in \mathcal{B}_{S} \otimes_{\text {alg }} \mathcal{T}$. The module number density $n(t, T)$ is defined by:

$$
n(t, T)=n\left(t, \sum_{j} c_{S, j} \otimes T_{j}\right):=\sum_{j} c_{S, j} \otimes n\left(t, T_{j}(t)\right) .
$$

Remark 2.24. We will use the following notations.

$$
b^{\varepsilon}(t, A)= \begin{cases}b(t, A), & \varepsilon=-1 ; A \in \mathcal{B}_{S} \otimes L^{2}\left(\mathbb{R}^{d}, \mathcal{K}\right) \\ b^{+}(t, A), & \varepsilon=+1 ; A \in \mathcal{B}_{S} \otimes L^{2}\left(\mathbb{R}^{d}, \mathcal{K}\right) \\ 1, & \varepsilon=0 ; A \in \mathcal{B}_{S} \\ n(t, A), & \varepsilon=2 ; A \hat{\in} \mathcal{B}_{S} \otimes \mathcal{T} .\end{cases}
$$

Let $T: t \in \mathbb{R}^{d} \rightarrow \mathcal{T} \hookrightarrow B\left(\mathcal{H}_{S} \otimes \mathcal{K}\right)$ be a strongly measurable map.

Definition 2.25. The number operator is defined by

$$
N(T)=\int n(t, T(t)) d t
$$

In particular:

$$
N_{t}\left(c_{S} \otimes T\right):=N\left(c_{S} \otimes \chi_{[0, t]} \otimes T\right)=c_{S} \otimes N_{t}\left(\chi_{[0, t]} \otimes T\right)
$$

Let recall the following well known fact [31]:

Proposition 2.26. For any $T, S \in \mathcal{B}_{S} \otimes \mathcal{T}$, we have:

$$
\begin{gathered}
N(T)^{*}=N\left(T^{*}\right) \\
N(S) N(T)=N(S T)
\end{gathered}
$$

Proposition 2.27. For each $t \in \mathbb{R}_{+}$, the map $T \in \mathcal{B}_{S} \otimes \mathcal{T} \mapsto N_{t}(T)$ is continuous from the strong operator topology on $\mathcal{B}\left(\mathcal{H}_{S} \otimes \mathcal{K}\right)$ to the topology of strong convergence on module exponential vectors whose test functions are finite step functions. 
Remark 2.28. By inspection of the proof below, it is clear that the condition that $T$ is bounded is not needed. In this case, some obvious domain conditions have to be introduced.

Proof. For any module exponential vector $\xi=\sum_{\alpha \in K} h_{S, \alpha} \otimes \psi_{\alpha} \in \mathcal{H}_{S} \otimes \Gamma\left(L^{2}\left(\mathbb{R}^{d}, \mathcal{K}\right)\right)$ with $\alpha \in L^{2}\left(\mathbb{R}^{d}, \mathcal{K}\right)$ a step function, for $T \in \mathcal{B}_{S} \otimes \mathcal{T}$ and $K$ a finite set, we have:

$$
\begin{aligned}
\left\|N_{t}(T) \xi\right\|^{2} & =\left\|N_{t}(T) \sum_{\alpha} h_{S, \alpha} \otimes \psi_{\alpha}\right\|^{2} \\
& =\sum_{\alpha_{1}, \alpha_{2}}\left\langle h_{S, \alpha_{1}},\left\langle\psi_{\alpha_{1}}, N_{t}(T)^{*} N_{t}(T) \psi_{\alpha_{2}}\right\rangle h_{S, \alpha_{2}}\right\rangle \\
& =\sum_{\alpha_{1}, \alpha_{2}}\left\langle h_{S, \alpha_{1}},\left\langle\psi_{\alpha_{1}}, N_{t}\left(T^{*}\right) N_{t}(T) \psi_{\alpha_{2}}\right\rangle h_{S, \alpha_{2}}\right\rangle \\
& =\sum_{\alpha_{1}, \alpha_{2}}\left\langle h_{S, \alpha_{1}},\left\langle\psi_{\alpha_{1}}, N_{t}\left(T^{*} T\right) \psi_{\alpha_{2}}\right\rangle h_{S, \alpha_{2}}\right\rangle \\
& =\sum_{\alpha_{1}, \alpha_{2}}\left\langle h_{S, \alpha_{1}},\left\langle\psi_{\alpha_{1}}, N_{t}\left(|T|^{2}\right) \psi_{\alpha_{2}}\right\rangle h_{S, \alpha_{2}}\right\rangle \\
& =\int_{0}^{t} \sum_{\alpha_{1}, \alpha_{2}}\left\langle h_{S, \alpha_{1}},\left\langle\alpha_{1}(t),|T|^{2} \alpha_{2}(t)\right\rangle h_{S, \alpha_{2}}\right\rangle\left\langle\psi_{\alpha_{1}}, \psi_{\alpha_{2}}\right\rangle d t
\end{aligned}
$$

By assumption, any $\alpha \in K$, has the form

$$
\alpha(s)=\sum_{\mu \in J} a_{\mu}^{(\alpha)} \chi_{I_{\mu}}(s) \alpha_{\mu}
$$

where $J$ is a finite set, $a_{\mu}^{(\alpha)} \in \mathbb{C}, \alpha_{\mu} \in K$ and $I_{\mu} \subseteq \mathbb{R}^{d}$ is a bounded set. The sets $J$ and $I_{\mu}$ generally will depend on $\alpha$ but, by taking refinements and remaining the $a_{\mu}^{(\alpha)}$ one can assume that these sets are independents of $\alpha \in K$. With these notations, one has:

$$
\begin{aligned}
& \left\|N_{t}(T) \xi\right\|^{2} \\
& \left.=\sum_{\mu \in J \alpha_{1}, \alpha_{2} \in K} \sum_{\alpha_{1}}, \psi_{\alpha_{2}}\right\rangle \overline{a_{\mu}^{\left(\alpha_{1}\right)}} a_{\mu}^{\left(\alpha_{2}\right)}\left|I_{\mu}\right|\left\langle T\left(h_{S, \alpha_{1}} \otimes \alpha_{1, \mu}\right), T\left(h_{S, \alpha 2} \otimes \alpha_{2, \mu}\right)\right\rangle
\end{aligned}
$$

where $\left|I_{\mu}\right|$ denotes the Lebesgue measure of $I_{\mu} \subseteq \mathbb{R}^{d}$. The (2.29) shows that, if $\overline{T_{n}}=$ $\sum_{j \in I_{n}} c_{S, j}^{(n)} \otimes T_{j}^{(n)}(t)$ converges to $T$ strongly on $\mathcal{H}_{S} \otimes \Gamma\left(L^{2}\left(\mathbb{R}^{d}, \mathcal{K}\right)\right)$, then $N_{t}\left(T_{n}\right)$ converges to $\left(N_{t}(T)\right)$ strongly on $\mathcal{E}$ where $\operatorname{Exp}_{0}\left(L^{2}\left(\mathbb{R}^{d}, \mathcal{K}\right)\right)$ is the algebraic linear span of the exponential vectors whose test functions are finite valued test functions. 


\section{Module commutation relations: the basic estimate}

Even in the case of a trivial Hilbert module, there is no simple closed form for the Boson module commutation relations. The identity proved in Proposition (3.1) however is sufficient to prove the module Itô table.

Proposition 3.1. For any $c_{S} \otimes f, d_{S} \otimes g \in \mathcal{B}_{S} \otimes L^{2}\left(\mathbb{R}^{d} ; \mathcal{K}\right)$, we have:

$$
\left[B\left(c_{S} \otimes f\right), B^{+}\left(d_{S} \otimes g\right)\right]=\left(c_{S} \otimes f \mid d_{S} \otimes g\right) \otimes 1+\left[c_{S}^{*}, d_{S}\right] \otimes B^{+}(g) B(f(\beta .1)
$$

Proof. Consider $c_{S} \otimes f, d_{S} \otimes g \in \mathcal{B}_{S} \otimes L^{2}\left(\mathbb{R}^{d} ; \mathcal{K}\right)$, using the fact that $\left[B(f), B^{+}(g)\right]=$ $\langle f, g\rangle 1_{\Gamma}$, we get:

$$
\begin{aligned}
& {\left[B\left(c_{S} \otimes f\right), B^{+}\left(d_{S} \otimes g\right)\right] } \\
= & {\left[c_{S}^{*} \otimes B(f), d_{S} \otimes B^{+}(g)\right] } \\
= & \left(c_{S}^{*} d_{S} \otimes B(f) B^{+}(g)-c_{S}^{*} d_{S} \otimes B^{+}(g) B(f)\right)+\left(c_{S}^{*} d_{S}-d_{S} c_{S}^{*}\right) \otimes B^{+}(g) B(f) \\
= & c_{S}^{*} d_{S} \otimes\langle f, g\rangle 1_{\Gamma}+\left[c_{S}^{*}, d_{S}\right] \otimes B^{+}(g) B(f) \\
= & \left(c_{S} \otimes f \mid d_{S} \otimes g\right) \otimes 1_{\Gamma}+\left[c_{S}^{*}, d_{S}\right] \otimes B^{+}(g) B(f)
\end{aligned}
$$

Proposition 3.2. For any $F, G \in \mathcal{B}_{S} \otimes L^{2}\left(\mathbb{R}^{d} ; \mathcal{K}\right)$, denoting

$$
\Delta:=\left[B(F), B^{+}(G)\right]-(F \mid G) \otimes 1
$$

One has, $\forall h_{1, S} \otimes \psi_{\varphi_{1}}, h_{2, S} \otimes \psi_{\varphi_{2}} \in \mathcal{E}$,

$$
\begin{aligned}
& \left|\left\langle h_{1, S} \otimes \psi_{\varphi_{1}}, \Delta h_{2, S} \otimes \psi_{\varphi_{2}}\right\rangle\right| \\
& \leq\left|\left\langle\left(1 \otimes \varphi_{2} \mid F\right) h_{1, S},\left(1 \otimes \varphi_{1} \mid G\right) h_{2, S}\right\rangle\left\langle\psi_{\varphi_{1}}, \psi_{\varphi_{2}}\right\rangle\right| \\
& \quad+\left|\left\langle\left(1 \otimes \varphi_{2} \mid F\right)^{*} h_{1, S},\left(1 \otimes \varphi_{1} \mid G\right)^{*} h_{2, S}\right\rangle\left\langle\psi_{\varphi_{1}}, \psi_{\varphi_{2}}\right\rangle\right|
\end{aligned}
$$

Proof. Let $F=\sum_{i} c_{S, i} \otimes f_{i}, \quad G=\sum_{j} d_{S, j} \otimes g_{j} \in \mathcal{B}_{S} \otimes L^{2}\left(\mathbb{R}^{d} ; \mathcal{K}\right)$. From Proposition (3.1), one knows that

$$
\Delta:=\left[B(F), B^{+}(G)\right]-(F \mid G) \otimes 1=\sum_{i} \sum_{j}\left(\left[c_{S, i}^{*}, d_{S, j}\right] \otimes B^{+}\left(g_{j}\right) B\left(f_{i}\right)\right)
$$


Therefore:

$$
\begin{aligned}
& \left|\left\langle h_{1, S} \otimes \psi_{\varphi_{1}}, \Delta h_{2, S} \otimes \psi_{\varphi_{2}}\right\rangle\right| \\
= & \left|\sum_{i} \sum_{j}\left\langle h_{1, S} \otimes \psi_{\varphi_{1}},\left[c_{S, i}^{*}, d_{S, j}\right] h_{2, S} \otimes B^{+}\left(g_{j}\right) B\left(f_{i}\right) \psi_{\varphi_{2}}\right\rangle\right| \\
= & \left|\sum_{i} \sum_{j}\left\langle h_{1, S},\left[c_{S, i}^{*}, d_{S, j}\right] h_{2, S}\right\rangle\left\langle\psi_{\varphi_{1}}, B^{+}\left(g_{j}\right) B\left(f_{i}\right) \psi_{\varphi_{2}}\right\rangle\right| \\
= & \mid \sum_{i} \sum_{j}\left(\left\langle c_{S, i} h_{1, S}, d_{S, j} h_{2, S}\right\rangle-\left\langle d_{S, j}^{*} h_{1, S}, c_{S, i}^{*} h_{2, S}\right\rangle\right) \\
& \times\left\langle B\left(g_{j}\right) \psi_{\varphi_{1}}, B\left(f_{i}\right) \psi_{\varphi_{2}}\right\rangle \mid \\
= & \mid \sum_{i} \sum_{j}\left(\left\langle c_{S, i} h_{1, S}, d_{S, j} h_{2, S}\right\rangle-\left\langle d_{S, j}^{*} h_{1, S}, c_{S, i}^{*} h_{2, S}\right\rangle\right) \\
& \times\left\langle\varphi_{1}, g_{j}\right\rangle\left\langle f_{i}, \varphi_{2}\right\rangle\left\langle\psi_{\varphi_{1}}, \psi_{\varphi_{2}}\right\rangle \mid \\
\leqslant & \left|\sum_{i} \sum_{j}\left\langle c_{S, i} h_{1, S}, d_{S, j} h_{2, S}\right\rangle\left\langle\varphi_{1}, g_{j}\right\rangle\left\langle f_{i}, \varphi_{2}\right\rangle\left\langle\psi_{\varphi_{1}}, \psi_{\varphi_{2}}\right\rangle\right| \\
& +\left|\sum_{i} \sum_{j}\left\langle d_{S, j}^{*} h_{1, S}, c_{S, i}^{*} h_{2, S}\right\rangle\left\langle\varphi_{1}, g_{j}\right\rangle\left\langle f_{i}, \varphi_{2}\right\rangle\left\langle\psi_{\varphi_{1}}, \psi_{\varphi_{2}}\right\rangle\right| \\
= & \left|\left\langle\left[\sum_{i} c_{S, i}\left\langle f_{i}, \varphi_{2}\right\rangle\right]\left[\sum_{j} d_{S, j}\left\langle\varphi_{1}, g_{j}\right\rangle\right] h_{1, S}, h_{2, S}\right\rangle\left\langle\psi_{\varphi_{1}}, \psi_{\varphi_{2}}\right\rangle\right| \\
& +\left|\left\langle\left[\sum_{i} c_{S, i}^{*}\left\langle f_{i}, \varphi_{2}\right\rangle\right]\left[\sum_{j} d_{S, j}^{*}\left\langle\varphi_{1}, g_{j}\right\rangle\right] h_{1, S}, h_{2, S}\right\rangle\left\langle\psi_{\varphi_{1}}, \psi_{\varphi_{2}}\right\rangle\right| \\
= & \left|\left\langle\left(1 \otimes \varphi_{2} \mid F\right) h_{1, S},\left(1 \otimes \varphi_{1} \mid G\right) h_{2, S}\right\rangle\left\langle\psi_{\varphi_{1}}, \psi_{\varphi_{2}}\right\rangle\right| \\
& +\mid\left\langle\left(1 \otimes \varphi_{2} \mid F\right)^{*} h_{1, S},\left(1 \otimes \varphi_{1}\left|G^{*} h_{2, S}\right\rangle\left\langle\psi_{\varphi_{1}}, \psi_{\varphi_{2}}\right\rangle \mid\right.\right.
\end{aligned}
$$

\section{Module Form of the Hudson-Parthasarathy Itô Table}

Let us recall the Hudson Parthasarathy-Itô-table [31]:

Proposition 4.1. Let $\mathcal{K}$ be a separable Hilbert space. Then, for any $f, g \in L^{2}(\mathbb{R} ; \mathcal{K})$, $S, T \in \mathcal{B}\left(L^{2}(\mathbb{R} ; \mathcal{K})\right)$, one has:

\begin{tabular}{|c|c|c|c|}
\hline & $d B_{t}(g)$ & $d N_{t}(T)$ & $d B_{t}^{+}(g)$ \\
\hline$d B_{t}(f)$ & 0 & $d B_{t}\left(T^{*} f\right)$ & $\langle f, g\rangle_{\mathcal{K}} d t$ \\
\hline$d N_{t}(S)$ & 0 & $d N_{t}(S T)$ & $d B_{t}^{+}(S g)$ \\
\hline$d B_{t}^{+}(f)$ & 0 & 0 & 0 \\
\hline
\end{tabular}


In the following, we will use the following well known facts [32].

Lemma 4.2. Let $\left(A_{n}\right)$ and $\left(B_{n}\right)$ be two sequences in a $\mathcal{B}_{S}$-Hilbert module, which converge respectively to $A$ and $B$, then the sequence $\left(A_{n} \mid B_{n}\right)$ converges to $(A \mid B)$ in $\mathcal{B}_{S}$.

Lemma 4.3. Let $\left(T_{n}\right)$ and $\left(C_{n}\right)$ be two sequences in $\mathcal{B}_{S} \otimes \mathcal{B}(\Gamma)$ which converge strongly on $\mathcal{H}_{S} \otimes L^{2}\left(\mathbb{R}^{d}, \mathcal{K}\right)$, respectively to $T$ and $C$. Suppose that the sequence $\left(\left\|T_{n}\right\|_{\mathcal{B}_{S} \otimes \mathcal{B}(\Gamma)}\right)$ is bounded. Then $N\left(T_{n} C_{n}\right)$ converges to $N(T C)$ strongly on $\mathcal{H}_{S} \otimes \operatorname{Exp}_{0}\left(L^{2}\left(\mathbb{R}^{d}, \mathcal{K}\right)\right)$.

Proof. The statement follows from Proposition (2.27) because under our assumptions $T_{n} C_{n}$ converges strongly to $T C$.

Lemma 4.4. Either $(i)$ the sequence $T_{n} \in \mathcal{B}\left(\mathcal{H}_{S} \otimes \Gamma\right)$ is bounded and $\xi_{n} \rightarrow 0$ in $\mathcal{B}_{S} \otimes \Gamma$ with respect the Hilbert module topology, or $T_{n} \rightarrow 0$ in the $\|\cdot\|_{\infty}$-norm on $\mathcal{B}\left(\mathcal{H}_{S} \otimes \Gamma\right)$ and $\xi_{n} \in \mathcal{B}_{S} \otimes \Gamma$, then \|\|$T_{n} \xi_{n}\left\|_{M}^{2}\right\|_{\infty} \rightarrow 0$.

Proof. Let $T_{n}$ and $\xi_{n}$ be sequences satisfying the above hypothesis, it follows:

$$
\left\|T_{n} \xi_{n}\right\|_{M}^{2}=\left(T_{n} \xi_{n} \mid T_{n} \xi_{n}\right)=\left(\xi_{n} \mid T_{n}^{*} T_{n} \xi_{n}\right)
$$

For any $h_{S}, h_{S}^{\prime} \in \mathcal{H}_{S}$

$$
\begin{aligned}
\left\langle h^{\prime}{ }_{S},\left(\xi_{n} \mid T_{n}^{*} T_{n} \xi_{n}\right) h_{S}\right\rangle= & \left\langle\xi_{n} h^{\prime}{ }_{S}, T_{n}^{*} T_{n}\left(\xi_{n} h_{S}\right)\right\rangle_{\mathcal{H}_{S} \otimes \Gamma} \\
& \leqslant\left\|T_{n}\right\|^{2}\left\langle\xi_{n} h^{\prime}{ }_{S}, \xi_{n} h_{S}\right\rangle_{\mathcal{H}_{S} \otimes \Gamma} \\
& =\left\|T_{n}\right\|^{2}\left\langle h^{\prime}{ }_{S},\left(\xi_{n} \mid \xi_{n}\right) h_{S}\right\rangle
\end{aligned}
$$

from this the statement follows.

Lemma 4.5. Let $\left(T_{n}\right)$ be a sequence in $\mathcal{B}_{S} \otimes \mathcal{B}(\Gamma)$ which converges in $\|\cdot\|_{\infty}$-norm to $T$ and $\left(A_{n}\right)$ be a sequence in $\mathcal{B}_{S} \otimes \Gamma$ which converges to $A$ with respect the Hilbert module topology, then the sequence $T_{n} A_{n}$ converges to $T A$ with respect the Hilbert module topology of $\mathcal{B}_{S} \otimes \Gamma$.

Proof. For any $\psi \in \mathcal{B}_{S} \otimes \Gamma$, we have

$$
\begin{aligned}
\left(T_{n} A_{n}-T A \mid \psi\right) & =\left(T_{n} A_{n}-T_{n} A \mid \psi\right)+\left(T_{n} A-T A \mid \psi\right) \\
& =\left(T_{n}\left(A_{n}-A\right) \mid \psi\right)+\left(T_{n} A-T A \mid \psi\right)
\end{aligned}
$$

Using Cauchy Schwartz inequality, this leads to

$$
\begin{aligned}
\left\|\left(T_{n} A_{n}-T A \mid \psi\right)\right\|_{\infty} \leqslant & \|\| T_{n}\left(A_{n}-A\right)\left\|_{M}\right\|_{\infty}\|\| \psi\left\|_{M}\right\|_{\infty} \\
& +\|\| T_{n} A-T A\left\|_{M}\right\|_{\infty}\|\| \psi\left\|_{M}\right\|_{\infty}
\end{aligned}
$$

which converges to 0 under the hypothesis. 
Proposition 4.6. Let $F, G, F_{1}, G_{1}$ be finite sets, $\left(e_{i}, i \geqslant 0\right)$ an orthonormal basis of $L^{2}\left(\mathbb{R}^{d}, \mathcal{K}\right)$ and $e_{i, j}=\left|e_{i}\right\rangle\left\langle e_{j}\right| \in \mathcal{B}\left(L^{2}\left(\mathbb{R}^{d}, \mathcal{K}\right)\right)$. Consider

$$
\begin{aligned}
& A=\sum_{i \in F} a_{i, 0} \otimes e_{i} \in \mathcal{B}_{S} \otimes_{a l g} L^{2}\left(\mathbb{R}^{d}, \mathcal{K}\right) \\
& B=\sum_{j \in G} a_{1, j} \otimes e_{j} \in \mathcal{B}_{S} \otimes_{a l g} L^{2}\left(\mathbb{R}^{d}, \mathcal{K}\right) \\
& T=\sum_{(i, j) \in F_{1} \times G_{1}} a_{i, j} \otimes e_{i, j} \in \mathcal{B}_{S} \otimes_{a l g} \mathcal{T} \\
& C=\sum_{(i, j) \in F_{2} \times G_{2}} a_{i, j}^{\prime} \otimes e_{i, j} \in \mathcal{B}_{S} \otimes_{a l g} \mathcal{T}
\end{aligned}
$$

Then,

$$
\begin{gathered}
d B_{t}(A) d B_{t}^{+}(B)=(A \mid B) d t \\
d B_{t}(A) d N_{t}(T)=d B_{t}\left(T^{*} A\right) \\
d N_{t}(T) d B_{t}^{+}(B)=d B_{t}^{+}(T B) \\
d N_{t}(T) d N_{t}(C)=d N_{t}(T C)
\end{gathered}
$$

Proof. Using the Itô table of Proposition 4.1, one finds

$$
\begin{aligned}
d B_{t}(A) d B_{t}^{+}(B) & =\left(\sum_{i \in F} a_{i, 0}^{*} \otimes d B_{t}\left(e_{i}\right)\right)\left(\sum_{j \in G} a_{0, j} \otimes d B_{t}^{+}\left(e_{j}\right)\right) \\
& =\sum_{(i, j) \in F \times G} a_{i, 0}^{*} a_{0, j} \otimes d B_{t}\left(e_{i}\right) d B_{t}^{+}\left(e_{j}\right) \\
& =\sum_{(i, j) \in F \times G} \delta_{i, j} a_{i, 0}^{*} a_{0, j} \otimes d t \\
& =(A \mid B) d t \\
d B_{t}(A) d N_{t}(T)= & \left(\sum_{i \in F} a_{i, 0}^{*} \otimes d B_{t}\left(e_{i}\right)\right)\left(\sum_{\left(i^{\prime}, j\right) \in F_{1} \times G_{1}} a_{i^{\prime}, j} \otimes d N_{t}\left(e_{i^{\prime}, j}\right)\right) \\
= & \sum_{\left(i, i^{\prime}, j\right) \in F \times F_{1} \times G_{1}} a_{i, 0}^{*} a_{i^{\prime}, j} \otimes d B_{t}\left(e_{i}\right) d N_{t}\left(e_{i^{\prime}, j}\right) \\
= & \sum_{\left(i, i^{\prime}, j\right) \in F \times F_{1} \times G_{1}} \delta_{i, i^{\prime}} a_{i, 0}^{*} a_{i^{\prime}, j} \otimes d B_{t}\left(e_{j}\right)=d B_{t}\left(T^{*} A\right)
\end{aligned}
$$




$$
\begin{aligned}
& d N_{t}(T) d B_{t}^{+}(B)=\left(\sum_{\left(i, j^{\prime}\right) \in F_{1} \times G_{1}} a_{i, j^{\prime}} \otimes d N_{t}\left(e_{i, j^{\prime}}\right)\right)\left(\sum_{i \in G} a_{1, j} \otimes d B_{t}^{+}\left(e_{j}\right)\right) \\
& =\sum_{\left(i, j, j^{\prime}\right) \in F_{1} \times G \times G_{1}} a_{i, j^{\prime}} a_{0, j} \otimes d N_{t}\left(e_{i, j^{\prime}}\right) d B_{t}^{+}\left(e_{j}\right) \\
& =\sum_{\left(i, j, j^{\prime}\right) \in F_{1} \times G \times G_{1}} \delta_{j, j^{\prime}} a_{i, j^{\prime}} a_{0, j} \otimes d N_{t}\left(e_{i, j^{\prime}}\right) d B_{t}^{+}\left(e_{j}\right) \\
& =\sum_{(i, j) \in F_{1} \times G \cap G_{1}} a_{i, j} a_{1, j} d B_{t}^{+}\left(e_{i}\right)=d B_{t}^{+}(T B) \\
& d N_{t}(T) d N_{t}(C)=\left(\sum_{(i, j) \in F_{1} \times G_{1}} a_{i, j} \otimes d N_{t}\left(e_{i, j}\right)\right)\left(\sum_{(u, v) \in F_{2} \times G_{2}} a_{u, v} \otimes d N_{t}\left(e_{u, v}\right)\right) \\
& =\sum_{(i, j, u, v) \in F_{1} \times G_{1} \times F_{2} \times G_{2}} a_{i, j} \cdot a_{u, v} d N_{t}\left(e_{i, j}\right) d N_{t}\left(e_{u, v}\right) \\
& =\sum_{(i, j, u, v) \in F_{1} \times G_{1} \times F_{2} \times G_{2}} a_{i, j} \cdot a_{u, v} d N_{t}\left(\left|e_{i}\right\rangle\left\langle e_{j}\right|\right) d N_{t}\left(\left|e_{u}\right\rangle\left\langle e_{v}\right|\right) \\
& =\sum_{(i, j, u, v) \in F_{1} \times G_{1} \times F_{2} \times G_{2}} a_{i, j} \cdot a_{u, v}\left\langle e_{j}, e_{u}\right\rangle d N_{t}\left(\left|e_{i}\right\rangle\left\langle e_{v}\right|\right) \\
& =\sum_{(i, j, u, v) \in F_{1} \times G_{1} \times F_{2} \times G_{2}} a_{i, j} \cdot a_{u, v} \delta_{j u} d N_{t}\left(e_{i, v}\right)=d N_{t}(T C)
\end{aligned}
$$

Corollary 4.7. Let be given $A, B$ be elements of the Hilbert module $\mathcal{B}_{S} \otimes L^{2}(\mathbb{R}, \mathcal{K})$ and let $T, C$ be bounded linear adjointable operators in $\mathcal{B}_{S} \otimes \mathcal{T}$. Consider the following convergent series with respect to the Hilbert module norms. Let

$$
\begin{gathered}
A=\sum_{i \in \mathbb{N}} a_{i, 0} \otimes e_{i} \\
B=\sum_{j \in \mathbb{N}} a_{0, j} \otimes e_{j} \\
T=\sum_{i, j \in \mathbb{N}} a_{i, j} \otimes e_{i, j} \\
C=\sum_{i, j \in \mathbb{N}} a_{i, j}^{\prime} \otimes e_{i, j}
\end{gathered}
$$

where $\left(e_{i}, i \geqslant 0\right)$ is an orthonormal basis of the separable Hilbert space $\mathcal{H}$ and $e_{i, j}=$ $\left|e_{i}\right\rangle\left\langle e_{j}\right|$. Let moreover $T$ and $C$ be of the form where the series converge strongly on 
$\mathcal{L}_{a}\left(\mathcal{B}_{S} \otimes L^{2}(\mathbb{R}, \mathcal{K})\right)$, then,

$$
\begin{gathered}
d B_{t}(A) d B_{t}^{+}(B)=(A \mid B) d t \\
d B_{t}(A) d N_{t}(T)=d B_{t}\left(T^{*} A\right) \\
d N_{t}(T) d B_{t}^{+}(B)=d B_{t}^{+}(T B) \\
d N_{t}(T) d N_{t}(C)=d N_{t}(T C)
\end{gathered}
$$

Proof. First, we apply Proposition (4.6), for the finite sums. Then, by using Lemma (4.2), (4.3) and (4.5), we pass to the limit using continuity and we get the result.

Definition 4.8. Let $\mathcal{B}_{1}=\mathcal{B}_{S} \otimes \mathcal{B}(\mathcal{K}), \mathcal{B}_{S}$ be $\mathbb{C}^{*}$-algebras and let $\mathcal{H}$ be a $\mathcal{B}_{S}$-Hilbert module with a left action of $\mathcal{B}_{1}$ on $\mathcal{H}$ (i.e. a $*$-representation of $\mathcal{B}_{1}$ into the linear operators on $\mathcal{H}$ ) denoted $T: \xi \in \mathcal{H} \rightarrow T \xi \in \mathcal{H} ; \quad T \in \mathcal{B}_{1}$. The right action of $\mathcal{B}_{1}$ on $\mathcal{H}^{*}$, the dual of $\mathcal{H}$ : The Itô algebra $\mathcal{I}\left(\mathcal{B}_{1}, \mathcal{H}, \mathcal{B}_{S}\right)$, associated to the triple $\left(\mathcal{B}_{1}, \mathcal{H}, \mathcal{B}_{S}\right)$ is the $*$-algebra of $2 \times 2$ matrices

$$
\left(\begin{array}{ll}
T & \xi \\
\eta^{*} & b
\end{array}\right) ; \quad T \in \mathcal{B}_{1}, \xi \in \mathcal{H}, \eta^{*} \in \mathcal{H}^{*}, b \in \mathcal{B}_{S}
$$

with the multiplication

$$
\left(\begin{array}{ll}
T_{1} & \xi_{1} \\
\eta_{1}^{*} & b_{1}
\end{array}\right)\left(\begin{array}{ll}
T_{2} & \xi_{2} \\
\eta_{2}^{*} & b_{2}
\end{array}\right):=\left(\begin{array}{ll}
T_{1} T_{2} & T_{1} \xi_{2} \\
\eta_{1}^{*} T_{2} & \eta_{1}^{*} \xi_{2}
\end{array}\right)
$$

and with involution

$$
\left(\begin{array}{cc}
T & \xi \\
\eta^{*} & b
\end{array}\right)^{*}=\left(\begin{array}{cc}
T^{*} & \eta \\
\xi^{*} & b^{*}
\end{array}\right)
$$

Remark 4.9. The associativity of the multiplication (4.1) can be easily checked.

One of the advantage of the Hilbert module representation, is the following isomorphism which will be used frequently to get the results in the sequel.

Proposition 4.10. The map

$$
d M_{t}:\left(\begin{array}{cc}
T & \xi \\
\eta^{*} & b
\end{array}\right) \rightarrow d M_{t}\left(\begin{array}{cc}
T & \xi \\
\eta^{*} & b
\end{array}\right):=d N_{t}(T)+d B_{t}^{+}(\xi)+d B_{t}(\eta)+d t(b)
$$

is a *-isomorphism from the Itô algebra $\mathcal{I}\left(\mathcal{B}_{1}, \mathcal{H}, \mathcal{B}_{S}\right)$ into the algebra of stochastic differentials with mutual quadratic variation as multiplication. 
Proof. Clearly, the map $d M_{t}$ is linear. The independence of the differentials ([12]) leads to the injectivity of this map. Using the module Itô table given in Corollary (4.7), we get that

$$
d M_{t}\left(\begin{array}{ll}
T_{1} & \xi_{1} \\
\eta_{1}^{*} & b_{1}
\end{array}\right) d M_{t}\left(\begin{array}{ll}
T_{2} & \xi_{2} \\
\eta_{2}^{*} & b_{2}
\end{array}\right)=d M_{t}\left(\begin{array}{ll}
T_{1} T_{2} & T_{1} \xi_{2} \\
\eta_{1}^{*} T_{2} & \eta_{1}^{*} \xi_{2}
\end{array}\right)
$$

and

$$
\left(\begin{array}{cc}
T & \xi \\
\eta^{*} & b
\end{array}\right)^{*}=\left(\begin{array}{cc}
T^{*} & \eta \\
\xi^{*} & b^{*}
\end{array}\right)
$$

which implies that $*$ is a $*$-homomorphism. In fact, a $*$-isomorphism into its range.

\section{Module form of the White noise Unitarity}

From now on, we will fix the dimension $d$ to be 1 . To study the module form of the white noise unitary condition and the white noise flows, it is convenient to identify any element of the trivial Hilbert module $\mathcal{B}_{S} \otimes L^{2}(\mathbb{R}, \mathcal{K})$ with the 1-particle sub-module of the trivial Hilbert module $\mathcal{B}_{S} \otimes \Gamma\left(L^{2}(\mathbb{R}, \mathcal{K})\right)$. This is done by associating, to any $\xi \in L^{2}(\mathbb{R}, \mathcal{K})$ the element $\breve{\xi} \in \Gamma\left(L^{2}(\mathbb{R}, \mathcal{K})\right)$ defined by $\breve{\xi}:=(0, \xi, 0, \cdots)$ and to any $A=\sum_{i} a_{i} \otimes \xi_{i} \in \mathcal{B}_{S} \otimes L^{2}(\mathbb{R}, \mathcal{K})$ the element $\check{A}=\sum_{i} a_{i} \otimes \check{\xi}_{i} \in \mathcal{B}_{S} \otimes \Gamma\left(L^{2}(\mathbb{R}, \mathcal{K})\right)$.

In the following, we will use the same notation for $A$ and $\check{A}$. Consider the following module white noise differential equation:

$$
d U_{t}=\left(b d t+d B_{t}(\eta)+d B_{t}^{+}(\xi)+d N_{t}(T)\right) U_{t} ; \quad U_{0}=1
$$

with adjoint

$$
d U_{t}^{*}=U_{t}^{*}\left(b^{*} d t+d B_{t}^{+}(\eta)+d B_{t}(\xi)+d N_{t}\left(T^{*}\right)\right) ; \quad U_{0}^{*}=1
$$

where $\eta, \xi, T, b$ are as in Proposition (4.10). Using the notation (4.3), (5.1) can be written as:

$$
d U_{t}=d M_{t}\left(\begin{array}{cc}
T & \xi \\
\eta^{*} & b
\end{array}\right) U_{t}
$$

with adjoint given by:

$$
d U_{t}^{*}=U_{t}^{*} d M_{t}\left(\begin{array}{cc}
T^{*} & \eta \\
\xi^{*} & b^{*}
\end{array}\right)
$$

In the following we will use the well known independence of the stochastic differential over the past filtration (see [31]). 
Theorem 5.1. The solution of the module quantum stochastic differential equation (5.1) is unitary if and only if

$$
\begin{cases}T^{*}+T+T T^{*}= & 0 \\ \xi+\eta+T \eta= & 0 \\ \eta^{*}+\xi^{*}+\eta^{*} T^{*}= & 0 \\ b+b^{*}+\eta^{*} \eta= & 0\end{cases}
$$

and

$$
\begin{cases}T^{*}+T+T^{*} T= & 0 \\ \xi^{*}+\eta^{*}+\xi^{*} T= & 0 \\ \eta+\xi+T^{*} \xi= & 0 \\ b+b^{*}+\xi^{*} \xi= & 0\end{cases}
$$

Proof. The co-isometry condition $U_{t}^{*} U_{t}=1$ is equivalent to:

$$
d\left(U_{t} U_{t}^{*}\right)=d\left(U_{t}\right) U_{t}^{*}+U_{t} d\left(U_{t}^{*}\right)+d\left(U_{t}\right) d\left(U_{t}^{*}\right)=0
$$

and the isometry condition to:

$$
d\left(U_{t}^{*} U_{t}\right)=d\left(U_{t}^{*}\right) U_{t}+U_{t}^{*} d\left(U_{t}\right)+d\left(U_{t}^{*}\right) d\left(U_{t}\right)=0
$$

Condition (5.7) is equivalent to:

$$
\begin{gathered}
0=\left(d M_{t}\left(\begin{array}{ll}
T & \xi \\
\eta^{*} & b
\end{array}\right)+d M_{t}\left(\begin{array}{cc}
T^{*} & \eta \\
\xi^{*} & b^{*}
\end{array}\right)+d M_{t}\left(\begin{array}{cc}
T & \xi \\
\eta^{*} & b
\end{array}\right)\left(\begin{array}{cc}
T^{*} & \eta \\
\xi^{*} & b^{*}
\end{array}\right)\right) U_{t} U_{t}^{*} \\
=\left(d M_{t}\left(\begin{array}{ll}
T & \xi \\
\eta^{*} & b
\end{array}\right)+d M_{t}\left(\begin{array}{cc}
T^{*} & \eta \\
\xi^{*} & b^{*}
\end{array}\right)+d M_{t}\left(\begin{array}{cc}
T T^{*} & T \eta \\
\eta^{*} T^{*} & \eta^{*} \eta
\end{array}\right)\right) U_{t} U_{t}^{*}
\end{gathered}
$$

Then, we get, using the independence of the basic integrator over the past;

$$
\left(\begin{array}{cc}
T+T^{*}+T T^{*} & B+A+T A \\
\eta^{*}+\xi^{*}+\eta^{*} T^{*} & b+b^{*}+\eta^{*} \eta
\end{array}\right)=0
$$

which is equivalent to (5.5). Similarly (5.8) leads to

$$
\begin{aligned}
& \left(\begin{array}{cc}
T^{*} & \eta \\
\xi^{*} & b^{*}
\end{array}\right)+\left(\begin{array}{cc}
T & \xi \\
\eta^{*} & b
\end{array}\right)+\left(\begin{array}{cc}
T^{*} & \eta \\
\xi^{*} & b^{*}
\end{array}\right)\left(\begin{array}{ll}
T & \xi \\
\eta^{*} & b
\end{array}\right) \\
& =\left(\begin{array}{cc}
T^{*} & \eta \\
\xi^{*} & b^{*}
\end{array}\right)+\left(\begin{array}{cc}
T & \xi \\
\eta^{*} & b
\end{array}\right)+\left(\begin{array}{ll}
T^{*} T & T^{*} \xi \\
\xi^{*} T & \xi^{*} \xi
\end{array}\right)=0
\end{aligned}
$$

which is equivalent to (5.6). 
Corollary 5.2. The stochastic equation (5.1) with bounded module coefficients has a unitary solution if and only if its coefficient matrix has the form:

$$
\left(\begin{array}{ll}
T & \xi \\
\eta^{*} & b
\end{array}\right)=\left(\begin{array}{cc}
W-1 & -W \eta \\
\eta^{*} & -\frac{1}{2} \eta^{*} \eta+i H
\end{array}\right)
$$

where $W$ is an unitary operator in $\mathcal{B}_{1}=\mathcal{B}_{S} \otimes \mathcal{B}(\mathcal{K})$ and $H$ is a self adjoint operator in $\mathcal{B}_{S}$.

Proof. The conditions

$$
\begin{aligned}
& T^{*}+T+T^{*} T=0 \\
& T^{*}+T+T T^{*}=0
\end{aligned}
$$

imply that $T$ is a differential unitary in the sense of ([11]), so $T=W-1$ where $W$ is unitary in $\mathcal{B}_{1}$. Moreover, the equation $\eta^{*}+\xi^{*}+\eta^{*} T^{*}=0$ leads to $\xi=-W \eta$.

\section{Module form of the flow equation}

\subsection{Stochastic derivations}

In the notations of Definition (4.8), we choose $\mathcal{H}$ to be the trivial $\mathcal{B}_{S}$-Hilbert module: $\mathcal{H}:=\left(\mathcal{B}_{S} \otimes \mathcal{K}\right)$ and $\mathcal{B}_{1}:=\mathcal{B}_{S} \otimes \mathcal{B}(\mathcal{K})$ with its action on $\mathcal{H}$ given by (2.6). Thus, we identify $\mathcal{B}_{S}$ to a $*$-sub-algebra of the $*$-algebra of linear adjointable operators on $\mathcal{H}$. The right action of $\mathcal{B}_{1}$ on the dual module $\left(\mathcal{B}_{S} \otimes \mathcal{K}\right)^{*}$ is defined by (2.7). The Itô algebra $\mathcal{I}\left(\mathcal{B}_{1}, \mathcal{H}, \mathcal{B}_{S}\right)$, is defined by Definition (4.8).

Definition 6.1. In these notations, the right action of $\mathcal{B}_{S}$ on $\mathcal{H}$ and on $\mathcal{H}^{*}$ and the natural right actions of $\mathcal{B}_{S}$ on itself and on $\mathcal{B}_{1}=\mathcal{B}_{S} \otimes \mathcal{B}(\mathcal{K})$ induces the right action $r($.$) on the Itô- algebra \mathcal{I}\left(\mathcal{B}_{1}, \mathcal{H}, \mathcal{B}_{S}\right)$ defined by

$$
\left(\begin{array}{cc}
T & \xi \\
\eta^{*} & b
\end{array}\right) r(x):=\left(\begin{array}{cc}
T x & \xi x \\
\eta^{*} x & b x
\end{array}\right)
$$

Similarly the corresponding left actions of $\mathcal{B}_{S}$ induce the left action $l($.$) of \mathcal{B}_{S}$ on $\mathcal{I}\left(\mathcal{B}_{1}, \mathcal{H}, \mathcal{B}_{S}\right)$, defined by:

$$
l(x)\left(\begin{array}{cc}
T & \xi \\
\eta^{*} & b
\end{array}\right):=\left(\begin{array}{cc}
x T & x \xi \\
x \eta^{*} & x b
\end{array}\right)
$$

where $T \in \mathcal{B}_{S} \otimes B_{\mathcal{K}}, \xi \in \mathcal{B}_{S} \otimes \mathcal{K}, \eta^{*} \in\left(\mathcal{B}_{S} \otimes \mathcal{K}\right)^{*}$ and $x \in \mathcal{B}_{S}$.

Definition 6.2. Let $\mathcal{I}$ be an algebra not necessarily with identity and let $l$ and $r$ be a left and a right action of $\mathcal{B}_{S}$ on $\mathcal{I}$ related by the identity

$$
(l(b) x)^{*}=x^{*} r\left(b^{*}\right) ; \forall b \in \mathcal{B}_{S}, x \in \mathcal{I}
$$


A stochastic derivation on $\mathcal{I}$ is a linear map $\delta: \mathcal{B}_{S} \rightarrow \mathcal{I}$ satisfying

$$
\delta(x y)=\delta(x) l(y)+r(x) \delta(y)+\delta(x) \delta(y) ; \forall x, y \in \mathcal{B}_{S}
$$

If $\delta$ satisfies $\delta\left(x^{*}\right)=\delta(x)^{*} \forall x \in \mathcal{B}_{S}$, then it is called a stochastic $*$-derivation.

In the following, we will use the notation $\delta(x) l(y)=\delta(x) y$ and $r(x) \delta(y)=x \delta(y)$.

Proposition 6.3. Let be given the linear maps:

$$
\begin{gathered}
\delta_{2}: \mathcal{B}_{S} \longrightarrow\left(\mathcal{B}_{S} \otimes \mathcal{B}(\mathcal{K})\right) \\
\delta_{1}: \mathcal{B}_{S} \longrightarrow \mathcal{B}_{S} \otimes \mathcal{K} \\
\delta_{-1}: \mathcal{B}_{S} \longrightarrow\left(\mathcal{B}_{S} \otimes \mathcal{K}\right)^{*} \\
\delta_{0}: \mathcal{B}_{S} \longrightarrow \mathcal{B}_{S}
\end{gathered}
$$

and define the linear map:

$$
\delta: x \in \mathcal{B}_{S} \longrightarrow\left(\begin{array}{cc}
\delta_{2}(x) & \delta_{1}(x) \\
\delta_{-1}(x)^{*} & \delta_{0}(x)
\end{array}\right) \in \mathcal{I}
$$

The map $\delta$ is a stochastic $*$-derivation if and only if for any $x, y \in \mathcal{B}$, we have :

$$
\begin{gathered}
\delta_{2}\left(x^{*}\right)=\delta_{2}(x)^{*} \\
\delta_{-1}\left(x^{*}\right)^{*}=\delta_{1}(x)^{*} \\
\delta_{-1}(x)=\delta_{1}\left(x^{*}\right) \\
\delta_{0}\left(x^{*}\right)=\delta_{0}(x)^{*} \\
\\
\delta_{2}(x y)=\delta_{2}(x) y+x \delta_{2}(y)+\delta_{2}(x) \delta_{2}(y) \\
\delta_{1}(x y)=\delta_{1}(x) y+x \delta_{1}(y)+\delta_{2}(x) \delta_{1}(y) \\
\delta_{-1}^{*}(x y)=\delta_{-1}(x)^{*} y+x \delta_{-1}(y)^{*}+\delta_{-1}(x)^{*} \delta_{2}(y) \\
\delta_{0}(x y)=\delta_{0}(x) y+x \delta_{0}(y)+\left(\delta_{-1}(x)^{*} \mid \delta_{1}(y)\right)
\end{gathered}
$$

Proof. $\delta$ is a stochastic $*$-derivation if and only if for any $x, y \in \mathcal{B}_{S}$, we have :

$$
\delta(x y)=\delta(x) l(y)+r(x) \delta(y)+\delta(x) \delta(y)
$$

where $l$ and $r$ are the left and right action given in the Definition (6.1), and

$$
\delta\left(x^{*}\right)=\delta(x)^{*}
$$


(6.9) is equivalent to

$$
\begin{aligned}
\left(\begin{array}{cc}
\delta_{2}(x y) & \delta_{1}(x y) \\
\delta_{-1}(x y)^{*} & \delta_{0}(x y)
\end{array}\right)= & \left(\begin{array}{cc}
\delta_{2}(x) & \delta_{1}(x) \\
\delta_{-1}(x)^{*} & \delta_{0}(x)
\end{array}\right) y+x\left(\begin{array}{cc}
\delta_{2}(y) & \delta_{1}(y) \\
\delta_{-1}(y)^{*} & \delta_{0}(y)
\end{array}\right) \\
& +\left(\begin{array}{cc}
\delta_{2}(x) & \delta_{1}(x) \\
\delta_{-1}(x)^{*} & \delta_{0}(x)
\end{array}\right)\left(\begin{array}{cc}
\delta_{2}(y) & \delta_{1}(y) \\
\delta_{-1}(y)^{*} & \delta_{0}(y)
\end{array}\right) \\
= & \left(\begin{array}{cc}
\delta_{2}(x) y & \delta_{1}(x) y \\
\delta_{-1}(x)^{*} y & \delta_{0}(x) y
\end{array}\right)+\left(\begin{array}{cc}
x \delta_{2}(y) & x \delta_{1}(y) \\
x \delta_{-1}(y)^{*} & x \delta_{0}(y)
\end{array}\right) \\
& +\left(\begin{array}{cc}
\delta_{2}(x) \delta_{2}(y) & \delta_{2}(x) \delta_{1}(y) \\
\delta_{-1}(x)^{*} \delta_{2}(y) & \left(\delta_{-1}(x)^{*} \mid \delta_{1}(y)\right)
\end{array}\right)
\end{aligned}
$$

From this, writing (6.10) in matrix form the statement follows.

\subsection{Module form of the forward inner flow equation}

Consider the following forward flow equation

$$
d j_{t}(x)=d\left(U_{t}^{*} x U_{t}\right)=\left(d U_{t}^{*}\right) x U_{t}+U_{t}^{*} x\left(d U_{t}\right)+\left(d U_{t}^{*}\right) x\left(d U_{t}\right)
$$

where $U_{t}$ satisfies the following stochastic equation in the notations (2.27):

$$
d U_{t}=D_{\varepsilon} d B_{t}^{\varepsilon} U_{t}
$$

where

$$
D_{\varepsilon}= \begin{cases}\eta, & \varepsilon=-1 \\ \xi, & \varepsilon=+1 \\ b, & \varepsilon=0 \\ T, & \varepsilon=2\end{cases}
$$

The following proposition gives an example of (inner) stochastic derivation on the Itô algebra $\mathcal{I}\left(\mathcal{B}_{1}, \mathcal{H}, \mathcal{B}_{S}\right)$

Proposition 6.4. Under the unitary conditions (5.9), equation (6.11) can be written in the form:

$$
d j_{t}(x)=U_{t}^{*} d M_{t}\left(\begin{array}{cc}
{\left[W^{*}, x\right] W} & W^{*}[x, W \eta] \\
{\left[\eta^{*} W^{*}, x\right] W} & -\frac{1}{2}\{(\eta \mid \eta), x\}+i[H, x]+(W \eta \mid x W \eta)
\end{array}\right) U_{t}
$$


Proof. Equation (5.9) is equivalent to:

$$
\begin{aligned}
d j_{t}(x)= & U_{t}^{*} d M_{t}\left(\begin{array}{cc}
T^{*} & \eta \\
\xi^{*} & b^{*}
\end{array}\right) x U_{t}+U_{t}^{*} x d M_{t}\left(\begin{array}{ll}
T & \xi \\
\eta^{*} & b
\end{array}\right) U_{t} \\
& +U_{t}^{*} d M_{t}\left(\begin{array}{cc}
T^{*} & \eta \\
\xi^{*} & b^{*}
\end{array}\right) x d M_{t}\left(\begin{array}{cc}
T & \xi \\
\eta^{*} & b
\end{array}\right) U_{t} \\
= & U_{t}^{*} d M_{t}\left(\begin{array}{cc}
T^{*} x & \eta x \\
\xi^{*} x & b^{*} x
\end{array}\right) U_{t}+U_{t}^{*} d M_{t}\left(\begin{array}{cc}
x T & x \xi \\
x \eta^{*} & x b
\end{array}\right) U_{t} \\
& +U_{t}^{*} d M_{t}\left(\begin{array}{cc}
T^{*} x & \eta x \\
\xi^{*} x & b^{*} x
\end{array}\right) d M_{t}\left(\begin{array}{cc}
T & \xi \\
\eta^{*} & b
\end{array}\right) U_{t} \\
= & U_{t}^{*} d M_{t}\left(\begin{array}{cc}
T^{*} x & \eta x \\
\xi^{*} x & b^{*} x
\end{array}\right) U_{t}+U_{t}^{*} d M_{t}\left(\begin{array}{cc}
x T & x \xi \\
x \eta^{*} & x b
\end{array}\right) U_{t} \\
& +U_{t}^{*} d M_{t}\left(\begin{array}{cc}
T^{*} x T & T^{*} x \xi \\
\xi^{*} x T & \xi^{*} x \xi
\end{array}\right) U_{t} \\
= & U_{t}^{*} d M_{t}\left(\begin{array}{ll}
T^{*} x+x T+T^{*} x T & \eta x+x \xi+T^{*} x \xi \\
\xi^{*} x+x \eta^{*}+\xi^{*} x T & b^{*} x+x b+\xi^{*} x \xi
\end{array}\right) U_{t}
\end{aligned}
$$

Then, using the unitary conditions (5.9) one finds (6.14).

\subsection{Module form of the backward inner flow equations}

Consider the backward inner flow equation

$$
d j_{t}(x)=d\left(U_{t} x U_{t}^{*}\right)=d\left(U_{t}\right) x U_{t}^{*}+U_{t} x d\left(U_{t}^{*}\right)+d\left(U_{t}\right) x d\left(U_{t}^{*}\right)
$$

where $U_{t}$ satisfy the equations (6.12), (6.13).

Remark 6.5. From the proof of Proposition (6.6) below it will be clear that the main difference between the forward and the backward flow equations is that, in the latter case one has to extend the domain of definition of the basic integrators so to give a meaning to expressions such as (for example) $d B_{t}\left(\eta^{*} j_{t}(x)\right)$. This can easily be done using the factorability of the Fock representation.

Proposition 6.6. Under the unitary conditions (5.9), equation (6.15) can be written in the form:

$$
d j_{t}(x)=d M_{t}\left(\begin{array}{cc}
W j_{t}(x) W^{*}-j_{t}(x) & W\left[j_{t}(x), \eta\right] \\
{\left[\eta^{*}, j_{t}(x)\right] W^{*}} & -\frac{1}{2}\left\{(\eta \mid \eta), j_{t}(x)\right\}+i\left[H, j_{t}(x)\right]+\left(\eta \mid j_{t}(x) \eta\right)
\end{array}\right)
$$


Proof. equation (6.15) is equivalent to:

$$
d j_{t}(x)=D_{\varepsilon} d B_{t}^{\varepsilon} U_{t} x U_{t}^{*}+U_{t} x U_{t}^{*} D_{\varepsilon}^{*} d B_{t}^{\varepsilon+}+D_{\varepsilon} d B_{t}^{\varepsilon} U_{t} x U_{t}^{*} D_{\varepsilon}^{*} d B_{t}^{\varepsilon+}
$$

where $j_{t}(x)=U_{t} x U_{t}^{*} \in \mathcal{B}_{S} \otimes \mathcal{B}_{\Gamma_{[0, t]}}\left(\right.$ where $\Gamma_{[0, t]}=\Gamma\left(L^{2}([0, t], \mathcal{K})\right)$, see [31] for more detail), satisfies

$$
j_{t}(x)=\sum_{\alpha} b_{S, \alpha} \otimes v_{t], \alpha}
$$

where $b_{S, \alpha} \in \mathcal{B}_{S}$ and $v_{t], \alpha} \in \mathcal{B}_{\Gamma_{[0, t]}}$. Then, using the adaptness property ([2]), it follows that for any $\xi=b_{S} \otimes \psi_{\mathcal{K}}$

$$
\begin{aligned}
d B_{t}(\xi) j_{t}(x) & =b_{S} \otimes B^{+}\left(\chi_{[t, t+d t]} \otimes \psi_{\mathcal{K}}\right)\left(\sum_{\alpha} b_{S, \alpha} \otimes v_{t], \alpha}\right) \\
& =\sum_{\alpha} b_{S} b_{S, \alpha} \otimes B^{+}\left(\chi_{[t, t+d t]} \otimes \psi_{\mathcal{K}}\right) \otimes v_{t], \alpha} \\
& =\sum_{\alpha} B^{+}\left(b_{S} b_{S, \alpha} \otimes \psi_{\mathcal{K}} \otimes \chi_{[t, t+d t]}\right) \otimes v_{t], \alpha}
\end{aligned}
$$

Using similar technique for the case $d B_{t}^{+}$and $d N_{t}$, it follows that:

$$
\begin{aligned}
d j_{t}(x)= & d M_{t}\left(\begin{array}{ll}
T & \xi \\
\eta^{*} & b
\end{array}\right) j_{t}(x)+j_{t}(x) d M_{t}\left(\begin{array}{ll}
T^{*} & \eta \\
\xi^{*} & b^{*}
\end{array}\right) \\
& +d M_{t}\left(\begin{array}{ll}
T & \xi \\
\eta^{*} & b
\end{array}\right) j_{t}(x) d M_{t}\left(\begin{array}{cc}
T^{*} & \eta \\
\xi^{*} & b^{*}
\end{array}\right) \\
= & d M_{t}\left(\begin{array}{cc}
T j_{t}(x) & \xi j_{t}(x) \\
\eta^{*} j_{t}(x) & b j_{t}(x)
\end{array}\right)+d M_{t}\left(\begin{array}{cc}
j_{t}(x) T^{*} & j_{t}(x) \eta \\
j_{t}(x) \xi^{*} & j_{t}(x) b^{*}
\end{array}\right) \\
& +d M_{t}\left(\begin{array}{cc}
T & \xi \\
\eta^{*} & b
\end{array}\right) d M_{t}\left(\begin{array}{ll}
j_{t}(x) T^{*} & j_{t}(x) \eta \\
j_{t}(x) \xi^{*} & j_{t}(x) b^{*}
\end{array}\right) \\
= & d M_{t}\left(\begin{array}{cc}
T j_{t}(x) & \xi j_{t}(x) \\
\eta^{*} j_{t}(x) & b j_{t}(x)
\end{array}\right)+d M_{t}\left(\begin{array}{cc}
j_{t}(x) T^{*} & j_{t}(x) \eta \\
j_{t}(x) \xi^{*} & j_{t}(x) b^{*}
\end{array}\right) \\
& +d M_{t}\left(\begin{array}{cc}
T j_{t}(x) T^{*} & T j_{t}(x) \eta \\
\eta^{*} j_{t}(x) T^{*} & \eta^{*} j_{t}(x) \eta
\end{array}\right) \\
= & d M_{t}\left(\begin{array}{ll}
T j_{t}(x)+j_{t}(x) T^{*}+T j_{t}(x) T^{*} & \xi j_{t}(x)+j_{t}(x) \eta+T j_{t}(x) \eta \\
\eta^{*} j_{t}(x)+j_{t}(x) \xi^{*}+\eta^{*} j_{t}(x) T^{*} & b j_{t}(x)+j_{t}(x) b^{*}+\eta^{*} j_{t}(x) \eta
\end{array}\right)
\end{aligned}
$$

Then, using the unitary conditions (5.9), we find (6.16). 


\subsection{Backward module flows}

In this section we consider the following backward equation:

$$
\begin{gathered}
d j_{t}(x)=d M_{t}\left(\begin{array}{cc}
\delta_{2}\left(j_{t}(x)\right) & \delta_{1}\left(j_{t}(x)\right) \\
\delta_{-1}\left(j_{t}(x)\right)^{*} & \delta_{0}\left(j_{t}(x)\right)
\end{array}\right)=: d M_{t}\left(\delta\left(j_{t}(x)\right)\right) \\
j_{0}(x)=x \quad \forall x \in B\left(\mathcal{H}_{S}\right)
\end{gathered}
$$

where the maps $\delta_{\varepsilon}$ are as in Proposition (6.3) (in particular they are bounded) and where the meaning of the right hand side of (6.18) has been explained in the proof of Proposition (6.6).

Theorem 6.7. The unique solution of the backward flow equation (6.18) is an identity preserving $*$-homomorphism if and only if the map

$$
\delta:=\left(\begin{array}{ll}
\delta_{2} & \delta_{1} \\
\delta_{1} & \delta_{0}
\end{array}\right)
$$

is a stochastic derivation.

Proof. The condition $j_{t}(1)=1$ is equivalent to $d j_{t}(1)=0$. The independence of the basic stochastic differentials shows that this is equivalent to:

$$
\delta_{2}(1)=\delta_{1}(1)=\delta_{0}(1)=\delta_{-1}(1)=0
$$

The condition: $j_{t}(x)^{*}=j_{t}\left(x^{*}\right)$ that the left hand of the two equations:

$$
\begin{gathered}
d j_{t}(x)^{*}=d M_{t}\left(\begin{array}{ll}
\delta_{2}\left(j_{t}(x)\right)^{*} & \delta_{-1}\left(j_{t}(x)\right) \\
\delta_{1}\left(j_{t}(x)\right)^{*} & \delta_{0}\left(j_{t}(x)\right)^{*}
\end{array}\right) \\
d j_{t}\left(x^{*}\right)=d M_{t}\left(\begin{array}{cc}
\delta_{2}\left(j_{t}\left(x^{*}\right)\right) & \delta_{1}\left(j_{t}\left(x^{*}\right)\right) \\
\delta_{-1}\left(j_{t}\left(x^{*}\right)\right)^{*} & \delta_{0}\left(j_{t}\left(x^{*}\right)\right)
\end{array}\right)
\end{gathered}
$$

are equal. By the independence of the basic integrators, this is equivalent to the four conditions (6.1), (6.2), (6.3), (6.4). Finally, equation (6.18) applied to $x y$ gives:

$$
d j_{t}(x y)=d M_{t}\left(\begin{array}{cc}
\delta_{2}\left(j_{t}(x y)\right) & \delta_{1}\left(j_{t}(x y)\right) \\
\delta_{-1}\left(j_{t}(x y)\right)^{*} & \delta_{0}\left(j_{t}(x y)\right)
\end{array}\right)
$$


The identity $j_{t}(x y)=j_{t}(x) j_{t}(y)$ is equivalent to the statement that the right hand side of equation (6.20) is equal to the right hand side of the following equation.

$$
\begin{aligned}
d j_{t}(x y)= & d j_{t}(x) j_{t}(y)+j_{t}(x) d j_{t}(y)+d j_{t}(x) d j_{t}(y) \\
= & d M_{t}\left(\begin{array}{cc}
\delta_{2}\left(j_{t}(x)\right) & \delta_{1}\left(j_{t}(x)\right) \\
\delta_{-1}\left(j_{t}(x)\right)^{*} & \delta_{0}\left(j_{t}(x)\right)
\end{array}\right) j_{t}(y) \\
& +j_{t}(x) d M_{t}\left(\begin{array}{cc}
\delta_{2}\left(j_{t}(y)\right) & \delta_{1}\left(j_{t}(y)\right) \\
\delta_{-1}\left(j_{t}(y)\right)^{*} & \delta_{0}\left(j_{t}(y)\right)
\end{array}\right) \\
& +d M_{t}\left(\begin{array}{cc}
\delta_{2}\left(j_{t}(x)\right) & \delta_{1}\left(j_{t}(x)\right) \\
\delta_{-1}\left(j_{t}(x)\right)^{*} & \delta_{0}\left(j_{t}(x)\right)
\end{array}\right) d M_{t}\left(\begin{array}{cc}
\delta_{2}\left(j_{t}(y)\right) & \delta_{1}\left(j_{t}(y)\right) \\
\delta_{-1}\left(j_{t}(y)\right)^{*} & \delta_{0}\left(j_{t}(y)\right)
\end{array}\right) \\
= & d M_{t}\left(\begin{array}{cc}
\delta_{2}\left(j_{t}(x)\right) & \delta_{1}\left(j_{t}(x)\right) \\
\delta_{-1}\left(j_{t}(x)\right)^{*} & \delta_{0}\left(j_{t}(x)\right)
\end{array}\right) j_{t}(y) \\
& +j_{t}(x) d M_{t}\left(\begin{array}{cc}
\delta_{2}\left(j_{t}(y)\right) & \delta_{1}\left(j_{t}(y)\right) \\
\delta_{-1}\left(j_{t}(y)\right)^{*} & \delta_{0}\left(j_{t}(y)\right)
\end{array}\right) \\
& +d M_{t}\left(\begin{array}{cc}
\delta_{2}\left(j_{t}(x)\right) \delta_{2}\left(j_{t}(y)\right) \\
\delta_{-1}\left(j_{t}(x)\right)^{*} \delta_{2}\left(j_{t}(y)\right) & \left(\delta_{-1}\left(j_{t}(x)\right)^{*} \mid \delta_{1}\left(j_{t}(y)\right)\right)
\end{array}\right) \\
= & d M_{t}\left(\begin{array}{cc}
\delta_{2}\left(j_{t}(x y)\right) & \delta_{1}\left(j_{t}(x y)\right) \\
\delta_{-1}\left(j_{t}(x y)\right)^{*} & \delta_{0}\left(j_{t}(x y)\right)
\end{array}\right)
\end{aligned}
$$

The independence of the basic integrators implies that this is equivalent to the identities (6.5), (6.6), (6.7), (6.8).

\subsection{Forward flow equation}

Theorem 6.8. Let $\delta_{2}, \delta_{-1}(.)^{*}, \delta_{1}, \delta_{0}$ be as in Theorem (6.7). The unique solution of the forward flow equation

$$
\begin{gathered}
d j_{t}(x)=j_{t}\left(d M_{t}\left(\begin{array}{cc}
\delta_{2}(x) & \delta_{1}(x) \\
\delta_{-1}(x)^{*} & \delta_{0}(x)
\end{array}\right)\right) \\
j_{0}(x)=x ; \quad \forall x \in B\left(\mathcal{H}_{S}\right)
\end{gathered}
$$

is an identity preserving $*-$ homomorphism if and only if the map (6.19) is a stochastic derivation.

Proof. The proof is based on the same idea as Theorem (6.7) and will be omitted. 


\section{Structure of stochastic derivation}

Theorem 7.1. Let $\mathcal{B}_{S}$ and $\hat{\mathcal{I}}$ be $*$-algebras and let $\delta$ be a linear map of $\mathcal{B}_{S}$ into $\hat{\mathcal{I}}$. Suppose that there exists $a *$-homomorphism $\imath: \mathcal{B}_{S} \longrightarrow \hat{\mathcal{I}}$, a right and left action

$$
\hat{r}: \mathcal{B}_{S} \longrightarrow \mathcal{L}(\hat{\mathcal{I}}), \quad \hat{l}: \mathcal{B}_{S} \longrightarrow \mathcal{L}(\hat{\mathcal{I}})
$$

satisfying the conditions

$$
\imath(b) x=\hat{l}(b) x, x \hat{r}(b)=x \imath(b) ; \forall b \in \mathcal{B}_{S} ; \forall x \in \hat{\mathcal{I}}
$$

Then the map

$$
\alpha:=\imath+\delta: \mathcal{B}_{S} \longrightarrow \mathcal{L}(\hat{\mathcal{I}})
$$

is $a *$-homomorphism if and only if $\delta$ satisfies the conditions:

$$
\begin{gathered}
\delta \text { is linear } \\
\delta(1)=0 \\
\delta\left(a^{*}\right)=\delta(a)^{*} \\
\delta(a b)=\delta(a) \hat{r}(b)+\hat{l}(a) \delta(b)+\delta(a) \delta(b)
\end{gathered}
$$

Proof. Notice that, if $\alpha$ and $\delta$ are related by (7.1), then

$$
\begin{gathered}
\alpha(a) \alpha(b)=(\imath(a)+\delta(a))(\imath(b)+\delta(b))=\imath(a b)+\hat{l}(a) \delta(b)+\delta(a) \hat{r}(b)+\delta(a) \delta(b) \\
\alpha(a b)=\imath(a b)+\delta(a b)
\end{gathered}
$$

So $\alpha$ is multiplicative if and only if $\delta$ satisfies (7.5). The remaining conditions are easily verified.

Corollary 7.2. In the notations of Theorem (6.7), the map $\delta_{2}$ has the form

$$
\delta(a)=\alpha(a)-\imath(a)
$$

where $\imath$ is the natural imbedding of $\mathcal{B}_{S}$ into $\mathcal{B}_{S} \otimes \mathcal{B}_{K}$ :

$$
\imath: b \in \mathcal{B}_{S} \longrightarrow b \otimes 1_{K} \in \mathcal{B}_{S} \otimes \mathcal{B}_{K}
$$

and $\alpha$ is a *-endomorphism of $\mathcal{B}_{S} \otimes \mathcal{B}_{K}$.

Proof. This follows from Theorem (7.1) with $\imath$ given by (7.6) and for any $b, b_{S} \in \mathcal{B}_{S}$, and $b_{K} \in \mathcal{B}_{K}$ :

$$
\hat{\mathcal{I}}=\mathcal{B}_{S} \otimes \mathcal{B}_{K} ; \hat{l}(b)\left(b_{S} \otimes b_{K}\right)=\left(b b_{S}\right) \otimes b_{K} ;\left(b_{S} \otimes b_{K}\right) \hat{r}(b)=\left(b_{S} b \otimes b_{K}\right)
$$


Remark 7.3. From the above results we define the following new form of the homomorphic flow equation.

Definition 7.4. In the notations of Definition (6.2), we denote $\mathcal{B}_{S} \oplus \mathcal{I}$ the $*$-algebra with product and involution given respectively by:

$$
\begin{gathered}
\left(b+a_{0}\right)\left(b^{\prime}+a_{0}^{\prime}\right):=b b^{\prime}+l(b) a_{0}^{\prime}+a_{0} r(b)+a_{0} a_{0}^{\prime} \in \mathcal{B}_{S} \oplus \mathcal{I} \\
\left(b+a_{0}\right)^{*}=b^{*}+a_{0}^{*}
\end{gathered}
$$

where $b, b^{\prime} \in \mathcal{B}_{S}, a_{0}, a_{0}^{\prime} \in \mathcal{I}$.

Remark 7.5. This algebra has no identity but the projection $1_{\mathcal{B}_{S}}+0$ acts as the identity on $\mathcal{B}_{S} \equiv \mathcal{B}_{S} \oplus 0$.

Corollary 7.6. In the notations of Definitions (6.2) and (7.4), any stochastic derivation $\delta$ in the sense of Definitions (6.2) has the form

$$
\delta(b)=\alpha(b)-b ; b \in \mathcal{B}_{S}
$$

where $\alpha$ is a*-homomorphism from $\mathcal{B}_{S}$ into $\mathcal{B}_{S} \oplus \mathcal{I}$ satisfying the condition

$$
\alpha(b)-b \in \mathcal{I}, \forall b \in \mathcal{B}_{S}
$$

Conversely, if $\alpha$ is a $*$-homomorphism from $\mathcal{B}_{S}$ into $\mathcal{B}_{S} \oplus \mathcal{I}$ satisfying conditions (7.8), then $\delta$, defined by (7.7) is a stochastic derivation.

Proof. Let $\hat{\mathcal{I}}:=\mathcal{B}_{S} \oplus \mathcal{I}$ and denote $\imath: b \in \mathcal{B}_{S} \longrightarrow \imath(b):=b+0_{\mathcal{I}}$ the natural embedding and define $\forall b, b_{S} \in \mathcal{B}_{S}, a_{0} \in \mathcal{I}$ :

$$
\begin{aligned}
& \hat{l}(b)\left(b_{S}+a_{0}\right):=b b_{S}+l(b) a_{0} \\
& \left(b_{S}+a_{0}\right) \hat{r}(b):=b_{S} b+a_{0} r(b)
\end{aligned}
$$

Then $\forall b, b_{S} \in \mathcal{B}_{S}, a_{0} \in \mathcal{I}$

$$
\begin{aligned}
& \imath(b)\left(b_{S}+a_{0}\right)=b b_{S}+l(b) a_{0}=\hat{l}(b)\left(b_{S}+a_{0}\right) \\
& \left(b_{S}+a_{0}\right) \imath(b)=b_{S} b+a_{0} r(b)=\left(b_{S}+a_{0}\right) \hat{r}(b)
\end{aligned}
$$

Then, given $\delta$, the map $\alpha$ defined by (7.7) is a $*$-homomorphism from $\mathcal{B}_{S}$ into $\mathcal{B}_{S} \oplus$ $\mathcal{I}$ by Theorem (7.1) and satisfies (7.8) by construction. Conversely, if $\alpha$ is a *homomorphism from $\mathcal{B}_{S}$ into $\mathcal{B}_{S} \oplus \mathcal{I}$ satisfying (7.8), then from Theorem (7.1) we know that the map

$$
\delta:=\alpha-\imath: \mathcal{B}_{S} \longrightarrow \mathcal{I}
$$

satisfies with $\hat{l}$ and $\hat{r}$ given by (7.9) and (7.10) respectively. From this it is easy to show that $\delta$ is a stochastic derivation in the sense of Definition (6.2).

Acknowledgments. I would like to thank X, Y and Z for their valuable comments on earlier drafts of this paper. 


\section{References}

1. L. Accardi, Quantum Probability: An Introduction to Some Basic Ideas and Trends, Modelos Estocasticos II 16, Sociedad Mathematica Mexicana, 2001.

2. L. Accardi, W. Ayed and H. Ouerdiane, White Noise Approach to Stochastic Integration. Random Operators and Stochastic Equations 13 (2005), 369-398.

3. L. Accardi, W. Ayed and H. Ouerdiane, White Noise Heisenbrg Evolution and EvansHudson Flows. To appear in Infinite Dimensional Analysis, Quantum Probability and Related Topics, (2006). Volterra preprint N. 590, (2005).

4. L. Accardi, Y. G. Lu, On the weak coupling limit for quantum electrodynamic. Proceeding Intern. Workshop of Math. Phys., SIENA F. Guerra, M. Loffredo (eds.) World Scientific (1992) 16-29 Volterra preprint N. 89 (1992).

5. L. Accardi, Y. G. Lu, The Wigner Semi-circle Law in Quantum Electro Dynamics. Comm. Math. Phys. 180 (1996), 605-632. Volterra preprint N. 126 (1992).

6. L. Accardi, Y. G. Lu and N. Obata, Towards a non-linear extension of stochastic calculus. In: Publications of the Research Institute for Mathematical Sciences, Kyoto, RIMS Kokyuroku 957, N. Obata, 1-15, (ed.) (1996).

7. L. Accardi, Y. G. Lu and I. Volovich, Quantum theory and its stochastic limit. Springer Verlag, 2002.

8. L. Accardi, Y. G. Lu and I. Volovich, The Stochastic Sector of Quantum Field Theory. Matematicheskie Zametki (1994). Volterra Preprint N. 138 (1993).

9. L. Accardi, Y. G. Lu and I. Volovich, Nonlinear extensions of classical and quantum stochastic calculus and essentially infinite dimensional analysis. In Probability Towards 2000; L. Accardi, Chris Heyde (eds.) Springer LN in Statistics 128 (1998), 1-33. Proceedings of the Symposium, Probability towards two thousand, Columbia University, New York, 2-6 October (1995).

10. L. Accardi, Y. G. Lu and I. Volovich, White noise approach to classical and quantum stochastic calculi. Lecture Notes of the Volterra-CIRM International School with the same title, Trento, Italy, 1999. Volterra Preprint N. 375 July (1999).

11. L. Accardi, A. Boukas, Unitarity conditions for the renormalized square of white noise. In Trends in Contemporary Infinite Dimensional Analysis and Quantum Probability, Natural and Mathematical Sciences Series 3, Italian School of East Asian Studies, Kyoto, Japan (2000) 7-36 Preprint Volterra N. 405 (2000).

12. L. Accardi and A. Boukas, Unitarity conditions for the renormalized square of white noise. Infinite Dimensional Anal. Quantum Probab. Related Topics 6 (2) (2003), 197-222. Preprint Volterra N. 466 (2001).

13. L. Accardi, F. Fagnola and J. Quaegebeur, A representation free Quantum Stochastic Calculus. Journ. Funct. Anal. 104 (1) (1992), 149-197.

14. L. Accardi, A. Boukas and U. Franz, Renormalized powers of quantum white noise. Inf. Dim. Anal. QPRT 8(4) (2005). 
15. L. Accardi, U. Franz and M. Skeide, Renormalized squares of white noise and other nonGaussian noises as Levy processes on real Lie algebras. Comm. Math. Phys. 228 (2002), 123-150. Preprint Volterra, N. 423 (2000).

16. L. Accardi, T. Hida and H-H. Kuo, The Itô table of the square of white noise. Infinite Dimensional Anal. Quantum Probab. Related Topics 4 (2001), 267-275. Preprint Volterra N. 459 (2001).

17. L. Accardi, R. L. Hudson, Quantum Stochastic Flows and non abelian Chomology. Volterra preprint N 16, (1989).

18. L. Accardi, M. Skeide, Realization of the square of white noise and the finite difference algebra in a Hilbert module, Mat. Zametki 68 (2000), 803-818.

19. V. P. Belavkin, A new form and $*$-algebraic structure of quantum stochastic integrals in Fock space. Rendiconti del Seminario Matematico e Fisico di Milano LVIII(1988), 177193.

20. M. Evans, R. L. Hudson, Multidimensional quantum diffusions. In Quantum Probability and Applications III. L. Accardi, W. von Waldenfels (eds.) Springer LNM 1303, 68-89, 1988.

21. D. Goswami, K. B. Sinha Hilbert modules and Stochastic Dilation of a Quantum Dynamical Semigroup on a von Neumann Algebra. Communication in Mathematical Physics, Springer-Verlag, N 205 (1999), 377-403.

22. D. Goswami, A. K. Pal and K. B. Sinha, Stochastic Dilation of a Quantum Dynamical Semgroup on a Separable Unital $C^{*}$ Algebra. Probability and Stochastics Series, CRC Press, Boca Raton, FL, (1996).

23. J. Gough, Non-commutative Ito and Stratonovich Noise and Stochastic Evolutions. Theoretical Math. Physics, 113 (1997), 276-284.

24. J. Gough,Noncommutative Markov approximations. Doklady Mathematics 64 (2001), 112-116. Dokl. Akad. Nauk, 379 (2001), 730-734. (in Russian)

25. R. L. Hudson, J. M. Lindsay, On Characterizing Quantum Stochastic Evolutions. Math. Proc. Cambridge Phil. Soc. 102 (1987), 363-370.

26. R. L. Hudson and K. R. Parthasarathy, Quantum Ito's formula and stochastic evolutions. Comm. Math. Phys., Communications in Mathematical Physics 93 (1984), 301-323.

27. H. H. Kuo, White noise distribution theory. Probability and Stochastics Series, CRC Press, Boca Raton, FL, 1996.

28. Lu Yun Gang, Quantum Stochastic Calculus on Hilbert Modules Driven by Poisson Processes. Volterra Preprint N 212, March (1995).

29. Lu Yun Gang, A note on free stochastic calculus on Hilbert modules and its applications. Random operators and Stoch. Equ. 4 (1996), 93-104. Volterra Preprint N 186, October (1994).

30. Lu Yun Gang, Quantum stochastic calculus, The passage from quantum system with continuous spectrum to quantum Poisson processes on Hilbert module. J. Math. Phys 36 (1995), 142-176. Volterra preprint N. 106 (1992). 
31. K. R. Parthasarathy, An introduction to quantum stochastic calculus. Monographs in Mathematics, 85, Birkhäuser Verlag, Basel, 1992.

32. S. Sakai, $\mathbb{C}^{*}$-algebras and $W^{*}$-algebra. Springer-Verlag Berlin Heidelberg, New York, 1971.

33. M. Skeide, Hilbert Module and Applications in Quantum Probability. Habilitation thesis, 2000.

34. M. Skeide, Quantum stochastic calculus on full Fock modules. Preprint, Rome, 1999 J. Funct. Anal.

35. R. Speicher, Combinatorial theory of the free product with amalgamation and operatorvalued free probability theory. Habilitation, Heidelberg (1994). Memoires of the American Mathematical Society 627 (1998).

36. T. Hida, Selected papers of Takeyuki Hida. Edited by L. Accardi, H. H. Kuo, N. Obata, K. Saitô, Si Si and L. Streit, World Scientific Publishing Co. Inc.

37. T. Hida, Brownian motion. Applications of Mathematics, 11, Translated from the Japanese by the author and T. P. Speed, Springer-Verlag, New York, 1980.

38. F. Trèves, Topological Vector Space, Distribution and Kernels. Academic Press, New York and London, 1967.

39. Yoshida, Kosaku, Functional analysis. Classics in Mathematics, Reprint of the sixth edition (1980). Springer-Verlag, Berlin, 1995.

Received 7 March, 2007; revised 13 July, 2007

\section{Author information}

L. ACCARDI, Centro Vito Volterra, Università di Roma "Tor Vergata", Via Columbia 2, 00133 Roma, Italy.

Email: accardi@volterra.mat.uniroma2.it

W. AYED, Institut Préparatoire aux Études d'Ingénieurs, El Merezka, 8000, Nabeul, Tunisia. Email: wided.ayed@ipein.rnu.tn

H. OUERDIANE, Department of mathematic Faculté des sciences de Tunis, 1060,, Tunis.

Email: mail:habib.ouerdiane.fst.rnu.tn 